\title{
A numerical model of the ionospheric signatures of time-varying magnetic reconnection: I. ionospheric convection
}

\author{
M. Lockwood ${ }^{1,2}$ and S. K. Morley ${ }^{2}$ \\ ${ }^{1}$ Rutherford Appleton Laboratory, Chilton, Oxfordshire, UK \\ ${ }^{2}$ Department of Physics and Astronomy, University of Southampton, Southampton, Hampshire, UK
}

Received: 9 September 2002 - Revised: 19 May 2003 - Accepted: 2 June 2003 - Published: 1 January 2004

\begin{abstract}
This paper presents a numerical model for predicting the evolution of the pattern of ionospheric convection in response to general time-dependent magnetic reconnection at the dayside magnetopause and in the cross-tail current sheet of the geomagnetic tail. The model quantifies the concepts of ionospheric flow excitation by Cowley and Lockwood (1992), assuming a uniform spatial distribution of ionospheric conductivity. The model is demonstrated using an example in which travelling reconnection pulses commence near noon and then move across the dayside magnetopause towards both dawn and dusk. Two such pulses, 8 min apart, are used and each causes the reconnection to be active for $1 \mathrm{~min}$ at every MLT that they pass over. This example demonstrates how the convection response to a given change in the interplanetary magnetic field (via the reconnection rate) depends on the previous reconnection history. The causes of this effect are explained. The inherent assumptions and the potential applications of the model are discussed.
\end{abstract}

Key words. Ionosphere (ionosphere-magnetosphere interactions; plasma convection) - Magnetospheric physics (magnetosphere-ionosphere interactions; solar windmagnetosphere interactions)

\section{Introduction}

Cowley and Lockwood (1992) developed a conceptual model of how ionospheric flow is excited on timescales shorter than the substorm cycle. Prior to this work, conceptual and empirical models of convection had been inherently steady-state in nature, in that they predicted a given pattern of ionospheric convection for a given prevailing orientation of the interplanetary magnetic field (IMF) (e.g. Cowley, 1984; Heelis et al., 1982; Heppner and Maynard, 1987; Holt et al., 1987; Hairston and Heelis, 1990; Reiff and Burch, 1985; Weimer, 1995). Such models did not consider the previous history

Correspondence to: M. Lockwood (m.lockwood@rl.ac.uk) of the IMF nor the phase of the substorm cycle. The same was true of the models of the corresponding patterns of ionospheric (Friis-Christensen et al., 1985) and field-aligned (Iijima et al., 1978; Erlandson et al., 1988) currents.

\subsection{Steady-state and time-varying convection}

Some fundamental principles of time-varying and steadystate electrodynamic ionosphere-magnetosphere coupling are illustrated in Fig. 1, from Lockwood and Cowley (1990). Figure 1a shows how a magnetopause reconnection $X$-line $A B$ maps down geomagnetic field lines to its ionospheric projection, a dayside merging gap $a b$. Similarly, an $X$-line $D E$ in the cross-tail current sheet maps to its projection $d e$ in the nightside ionosphere, and the "Stern gap" $C F$ maps to the polar cap diameter, $c f$. Consider the Faraday loop, fixed in space, $A B b a$ : Faraday's induction law, relating the electric field $E$ and the change in the magnetic field $B$ is, in integral form:

$\oint_{A B b a} \underline{E} \cdot \underline{d l}=\Phi_{A B}-\Phi_{a b}=\partial / \partial t \int_{A B b a} \underline{B} \cdot \underline{d a}$,

where $\underline{d l}$ is an element of length around the loop $A B b a$ and $\underline{d a}$ is an element of a surface area bounded by that loop. The left-hand side (LHS) of Eq. (1) is equal to the difference in voltages $\left(\Phi_{A B}-\Phi_{a b}\right)$ because two segments of the loop, $A a$ and $B b$, are field-aligned and, on large spatial scales at least, ideal-MHD applies and field-parallel electric fields are zero (i.e. $\underline{E} \cdot \underline{d l}=0$ for $A a$ and $B b$ ). In steady-state, all time derivatives are zero and thus $\Phi_{A B}=\Phi_{a b}$. In non-steady situations these voltages differ by the induction term on the right-hand side (RHS) of Eq. (1).

Similarly, application of the same logic to the loop $C F f c$ shows that the transpolar voltage $\Phi_{c f}$ equals the Stern gap voltage $\Phi_{C F}$ in steady state and application to the loop $D E e d$ shows that the nightside merging gap voltage $\Phi_{d e}$ equals the tail reconnection voltage $\Phi_{D E}$, again only under 


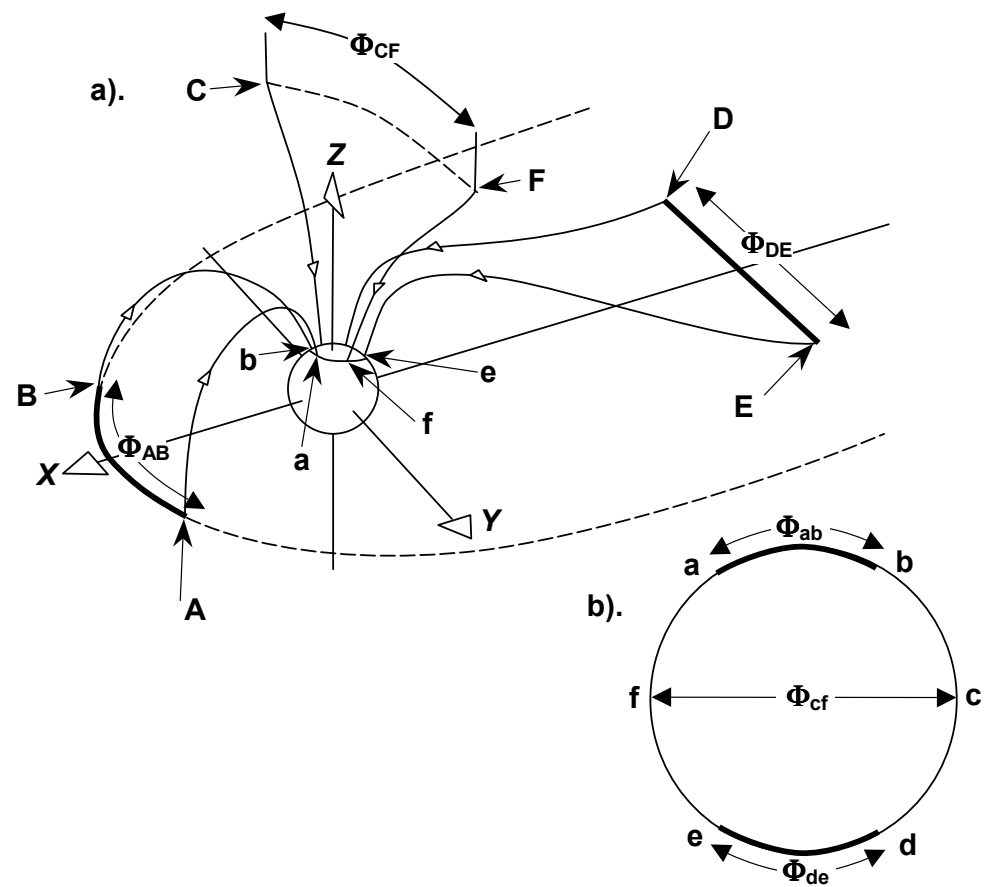

Fig. 1. Schematic of voltages in the magnetosphere generated by the solar wind flow and by magnetic reconnection. (a) A view of the magnetosphere from interplanetary space at northern mid-latitudes in the mid-afternoon sector, with the GSM axes marked. The dashed line is the equatorial magnetopause and the thick lines $\mathrm{AB}$ and $\mathrm{DE}$ are active reconnection sites in, respectively, the magnetopause and cross-tail current sheet. $C F$ is the Stern gap in interplanetary space. $A a, B b, C c, D d, E e$, and $F f$ are all magnetic field lines. (b) A view looking down on an ionospheric polar cap with 12:00 MLT to the top and 06:00 MLT to the right. The thin lines bd and ae are "adiaroic" (non-reconnecting) segments of the open-closed field line boundary (OCB), while $a b$ and $d e$ are the dayside and nightside merging gaps, the projections of the reconnection $X$-lines $A B$ and $D E$. The reconnection $X$-line voltages $\Phi_{A B}$ and $\Phi_{D E}$ are not, in general, equal to the corresponding merging gap voltages in the ionosphere, $\Phi_{a b}$ and $\Phi_{d e}$, because of induction effects. The polar cap voltage $\Phi_{c f}$ applies across the polar cap diameter cf which maps to the Stern gap, $C F$, across which the voltage is $\Phi_{C F}$.

steady conditions. Applying Faraday's law to the ionospheric polar cap boundary yields:

$$
\begin{aligned}
& \oint_{a b d e} \underline{E} \cdot \underline{d l}=\Phi_{a b}-\Phi_{d e}= \\
& \partial / \partial t \int_{a b d e} \underline{B_{i}} \cdot \underline{d a}=B_{i} \partial A_{p c} / \partial t .
\end{aligned}
$$

In this case, the non-reconnecting or "adiaroic" (meaning "not flowing across" - see Siscoe and Huang, 1985) segments of the open-closed boundary, $b d$ and $e a$, have zero boundary tangential electric field because they do not map to active reconnection $X$-lines zero (i.e. $\underline{E} \cdot \underline{d l}=0$ for $b d$ and $e a$ ) and thus the LHS of Eq. (2) equals the voltage difference $\left(\Phi_{a b}-\Phi_{d e}\right)$. Given that $\Phi_{a b}$ is the rate of flux transfer into the polar cap and $\Phi_{d e}$ is its rate of transfer out of the polar cap, Eq. (2) is effectively the continuity equation for the total open flux. The ionospheric field $B_{i}$ is constant to within a few percent (this is often referred to as the "ionospheric incompressibility" condition) and this allows derivation of the RHS of Eq. (2) which shows that departures from steady-state are manifested in changes of the polar cap area, $A_{p c}$. In steady state, the RHS of Eq. (2) is zero and $\Phi_{a b}=\Phi_{d e}$. Application of the same logic to the two halves of the polar cap bound by Faraday loops abcf and fcde yields that $\Phi_{a b}=\Phi_{d e}=\Phi_{c f}$ and thus, from above, $\Phi_{a b}=\Phi_{d e}=\Phi_{c f}=\Phi_{A B}=\Phi_{D E}=\Phi_{C F}$ under steadystate conditions. In this case, the electric field in interplanetary space maps spatially down the open field lines to give the polar cap electric field (Banks et al., 1984; Clauer and Banks, 1988) and all the voltages shown in Fig. 1 are the same.

However, Eqs. (1) and (2) show that induction terms, represented by the time derivatives, mean that these simplifications do not apply in time-dependent cases. For timescales too short for these steady-state concepts to apply, Cowley and Lockwood (1992) replaced the steady-state paradigm of the interplanetary electric field mapping spatially to the ionosphere with the concept of perturbation from zero-flow equilibria caused by time-dependent production $\left(\Phi_{A B}>\Phi_{D E}\right)$ or loss $\left(\Phi_{A B}<\Phi_{D E}\right)$ of the total open polar cap flux, $\left\{B_{i} A_{p c}\right\}$.

The solar wind flow causes newly-opened flux, produced by magnetic reconnection in the dayside magnetopause, to be appended to the tail lobe. The process is illustrated schematically in Fig. 2 by a series of noon-midnight cross-sections of the northern hemisphere of the magnetosphere. The area shaded grey in each part denotes newly-opened flux pro- 

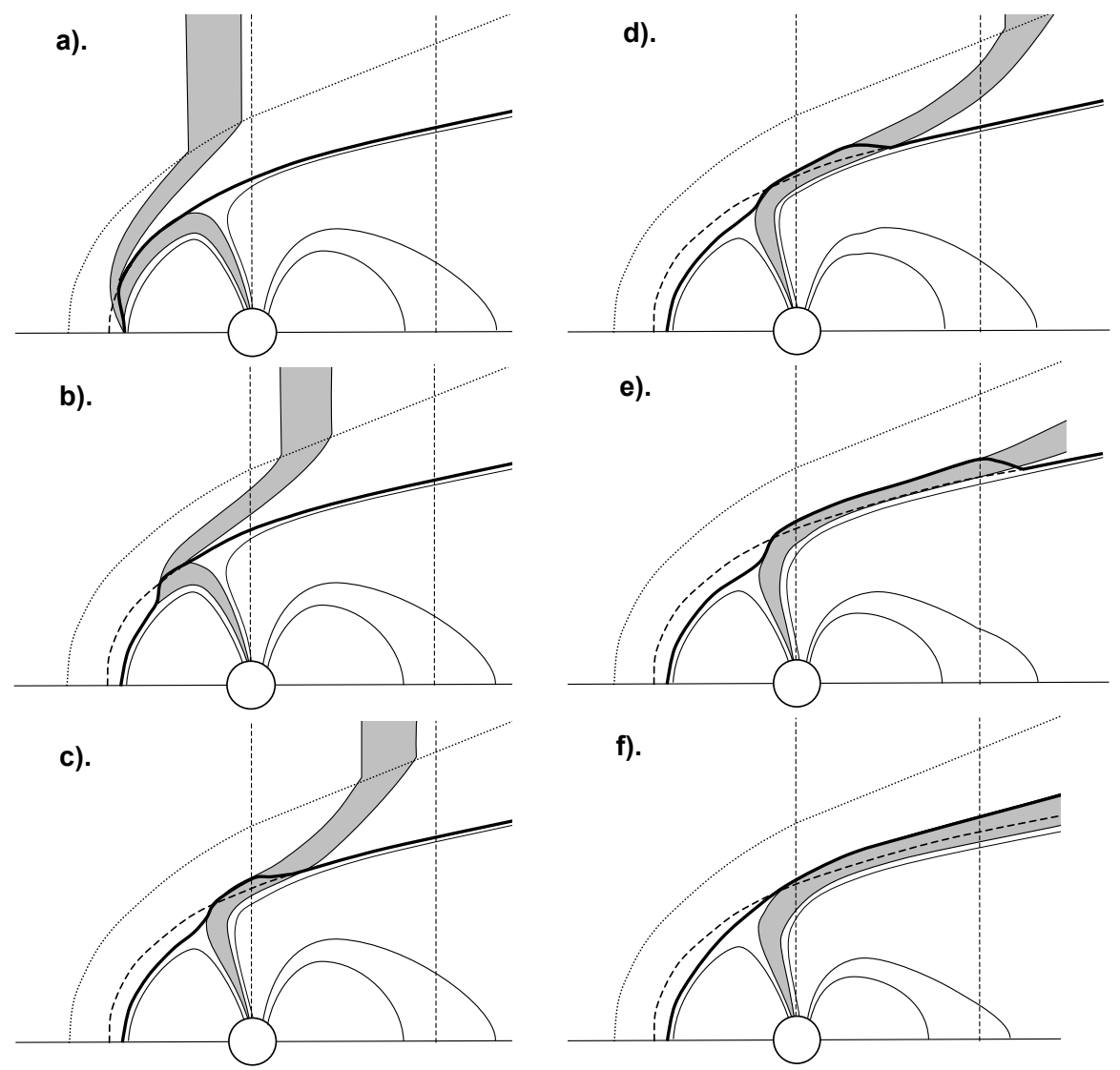

Fig. 2. Schematic noon-midnight cross-sections of the northern hemisphere magnetosphere, illustrating the evolution of newly-opened flux, shaded gray, as it is appended to the tail lobe by the solar wind flow following subsolar reconnection. The heavy solid line is the magnetopause current sheet, the dashed line shows its location prior to the reconnection pulse which produced the newly-opened flux. The dotted line is the bow shock and the two vertical lines are magnetospheric cross-sections at $[X]_{G S M}=0$ and $[X]_{G S M}=-30 R_{E}$. Thin lines are geomagnetic field lines.

duced by a pulse in the reconnection rate, in this case at an $X$-line at the nose of the magnetosphere. The vertical dashed lines show the $[Y Z]_{G S M}$ planes of the Geocentric Solar Magnetospheric coordinate system at $[X]_{G S M}=0$ and $[X]_{G S M}=-30 R_{E}$. In Fig. 2a, the reconnection pulse has just ended and the consequent erosion of the magnetosphere at the nose can be seen by comparing the current magnetopause location (heavy solid line) with its original, pre-pulse position (dashed line). In Fig. $2 \mathrm{~b}$ the antisunward evolution of the newly-opened flux, under the joint influence of the magnetic curvature force and the magnetosheath flow, can be seen, but the tail lobe at $[X]_{G S M}=0$ is not yet influenced. In Fig. $2 c$ the newly-opened flux has been appended to the tail lobe such that the magnetopause at $[X]_{G S M}=0$ has flared outward; however, the tail at $[X]_{G S M}=-30 R_{E}$ is yet to be affected. This does not happen until the time shown in Fig. 2e. Figure $2 \mathrm{f}$ shows the situation when the near-Earth magnetosphere has reached a new equilibrium with the new total open flux, with an eroded dayside and a flared tail (compare the final and initial magnetopause locations shown by the heavy solid and dashed lines).

The closed field lines in the near-Earth tail, shown in
Fig. 2, are influenced as the newly-opened field lines are appended to the tail. In the far tail, where the tail radius has reached its asymptotic limit, the undisturbed solar wind flow is parallel to the tail magnetopause and the solar wind dynamic pressure is not a factor. Thus the magnetic pressure in the tail lobe balances the static pressure in the solar wind (the sum of the thermal and magnetic pressures) and increasing the tail lobe flux increases the tail's cross-sectional area (i.e. the tail flares) but the magnetic pressure and field in the far-tail lobes remain constant. However, in the dayside and near-Earth tail, the solar wind dynamic pressure limits the magnetopause expansion and adding newly-opened flux increases the pressure in the near-Earth tail. In Figs. $2 a-c$, the tail pressure has yet to increase as the newly-opened flux has yet to reach that far antisunward. However in Figs. 2d-f, the increase in pressure is causing closed field lines in the tail to be compressed towards the cross-tail current sheet. This compression spreads down the tail as the newly-opened flux evolves antisunward.

Figure 3 presents a qualitative description of the convection patterns that result from the evolution of the newlyopened flux shown in Fig. 2. (It is the purpose of this pa- 

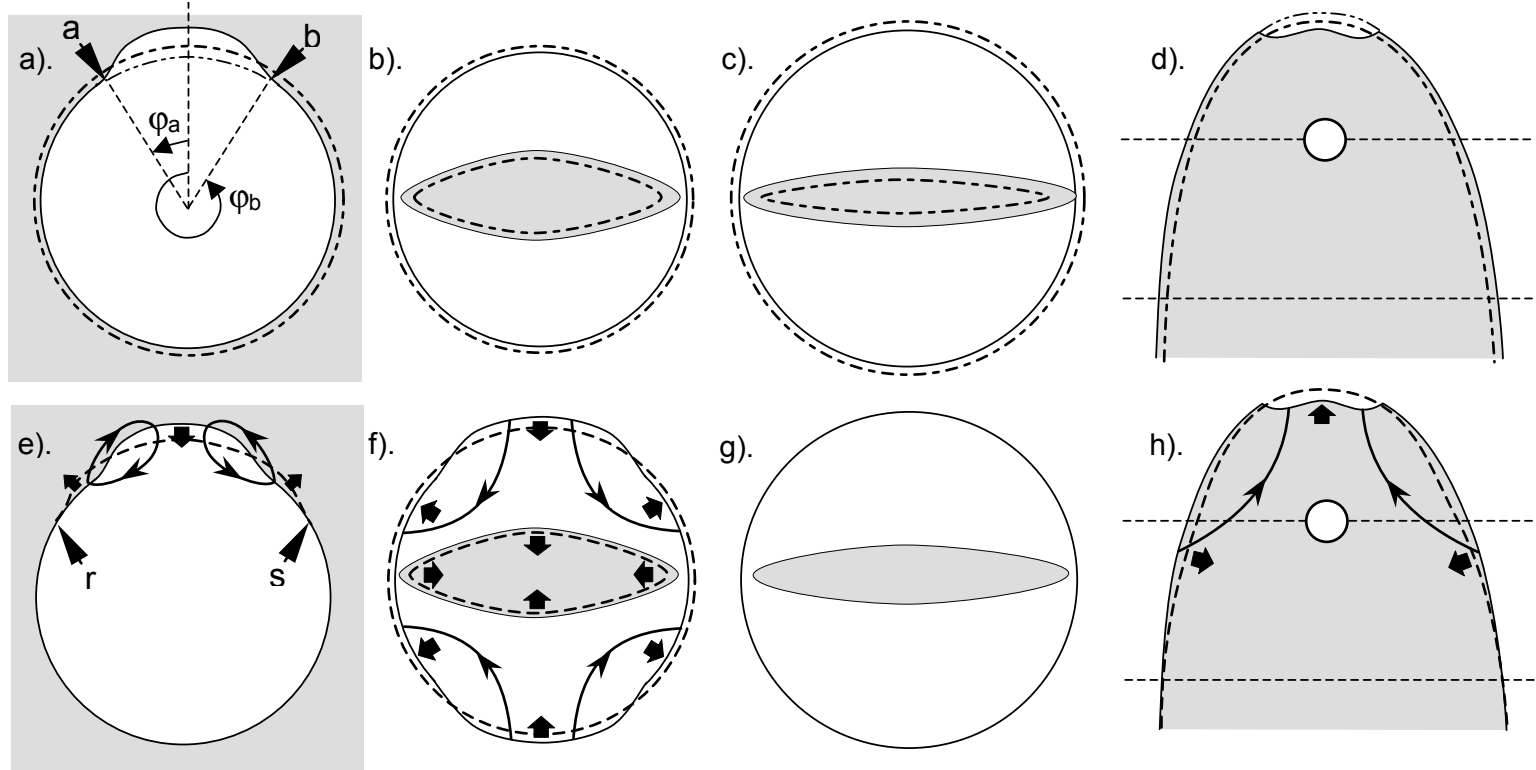

g).
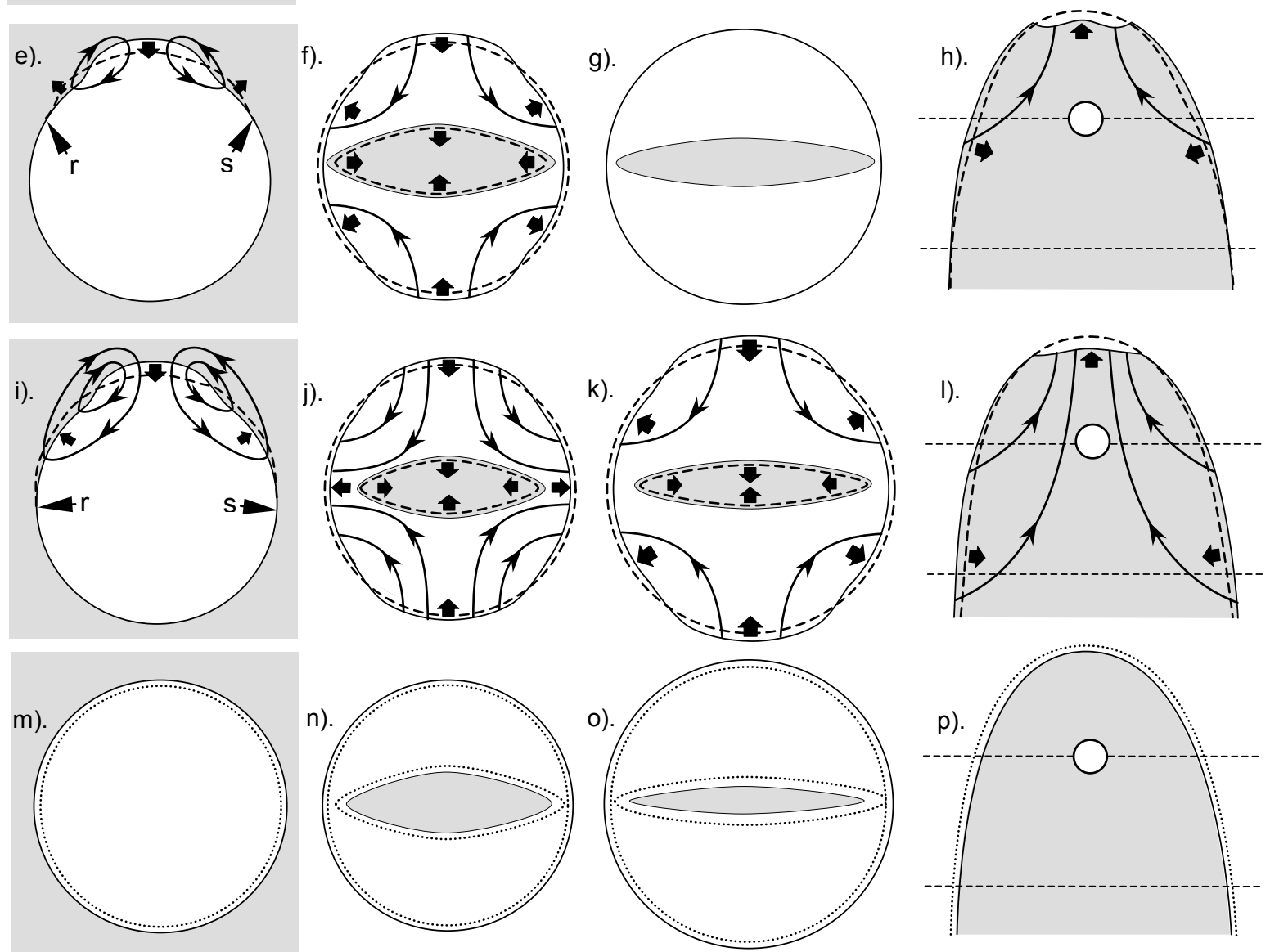

Fig. 3. Schematic illustration of the Cowley-Lockwood flow excitation model for the example of a single short-lived pulse of subsolar magnetopause reconnection which is completed by time $t=0$. The top row is for $t=0$, the second row for $t=4$ min, the third row for $t=14 \mathrm{~min}$ and the fourth row for $t>60 \mathrm{~min}$. The first column gives views of the ionospheric polar cap from above. The merging gap $\mathrm{ab}$ is marked in (a). The second and third columns show cross-sections of the magnetosphere at $[X]_{G S M}=0$ and $[X]_{G S M}=-30 R_{E}$, respectively, the solid line being the tail magnetopause. The fourth column gives views of the closed field line region in the equatorial plane of the magnetosphere with the $[X]_{G S M}$ axis up the page. The regions shaded grey are closed field lines, bounded by a solid line which is the open-closed boundary; lines with arrows are flow streamlines and the thick black arrows show boundary motions. In row 1 , the dot-dash lines give the final zero-flow equilibrium positions that the boundaries will eventually adopt (at $t$ greater than about 60 min). The double dot-dash lines in the top row show the boundary location before the reconnection pulse occurred. In rows 2 and 3 , the dashed lines show the equilibrium boundary positions for that instant of time. In row 4, the dotted lines show the boundary locations prior to the first reconnection pulse.

per to make the corresponding quantitative predictions). This schematic is a more detailed adaptation of that given by Cowley and Lockwood (1992). The four rows describe the situations at four different times relative to a single, short-lived pulse of magnetopause reconnection. The top row (Figs. 3ad) corresponds to Fig. 2a and is for time $t=0$, just after the reconnection pulse has taken place but before the ionospheric flow response has commenced. The second row $(3 \mathrm{e}-\mathrm{h})$ is for $t \approx 4 \mathrm{~min}$ and corresponds to Fig. 2c. Field lines evolve at speed $V_{F}$ over the magnetopause under the influence of magnetic tension and sheath flow. On the dayside, a constant $V_{F}$ of order $500 \mathrm{~km} \mathrm{~s}^{-1}$ would mean that the newly-opened 
field lines will have moved $19 R_{E}$ by $t=4 \mathrm{~min}$, sufficient to carry them to near $X=0$. The third row (Figs. 3i-l) is for $t \approx 14 \mathrm{~min}$. and corresponds to Fig. 2e. Gas dynamic modelling predicts that the sheath flow over the magnetopause $V_{s h}$ will average about $0.8 V_{s w}$ for $X$ between 0 and $-30 R_{E}$, where $V_{s w}$ is the speed of the undisturbed solar wind (Spreiter et al., 1966). Along the tail magnetopause we expect $V_{F} \approx V_{s h} \approx 0.8 V_{s w}$. From this we expect the newlyopened field lines to move tailward by about $30 R_{E}$ between $t=4$ and $t=14 \mathrm{~min}$. The fourth row (Figs. $3 \mathrm{~m}-\mathrm{p}$ ) is for $t$ greater than about $1 \mathrm{~h}$ when the effects of the reconnection pulse on convection have decayed away completely and correspond to Fig. 2f. The four columns in Fig. 3 show different parts of the coupled magnetosphere-ionosphere system and the lines with arrows are flow streamlines. The first column (parts a, e, i and $\mathrm{m}$ ) gives views of the northern hemisphere polar cap, with noon (12:00 MLT) to the top, 06:00 MLT to the right and 18:00 MLT to the left. The area shaded grey is the closed field line region, delineated by a solid line which is therefore the open-closed field line boundary (OCB). The second column ( $b, f, j$ and $n$ ) shows cross sections of the magnetosphere (parallel to the $[Y Z]_{G S M}$ plane) at $[X]_{G S M}=0$; the solid line is the magnetopause and the grey areas show closed field lines threading this tail cross section. The third column (c, $\mathrm{g}, \mathrm{k}$ and $\mathrm{o}$ ) is the same as column 2 for $[X]_{G S M}=-30 R_{E}$. Column $4(\mathrm{~d}, \mathrm{~h}, 1$ and $\mathrm{p}$ ) gives views of the closed field line region in the equatorial plane of the magnetosphere with $[X]_{G S M}$ up the page and $[Y]_{G S M}$ to the left. The horizontal dashed lines in column 4 show the locations of the cross sections given in columns 2 and 3 (and also as shown in Fig. 2).

Row one of Fig. 3 shows the locations of the magnetopause and the open-closed field line boundary immediately after a short-lived reconnection pulse has taken place, immediately after the Alfvén wave has arrived in the ionosphere but before any significant flow has begun (corresponding to Fig. 2a). The open flux in the tail lobe at this time is all "old", in that it was produced by prior periods of magnetopause reconnection which were sufficiently long before that the residual convection they cause in the near-Earth magnetosphere and ionosphere is negligible. The dashed-double-dot lines give the location of the dayside open-closed boundary just before the reconnection pulse and the dot-dash lines show the final zero-flow equilibrium locations of the open-closed and magnetopause boundaries. These zero-flow equilibrium boundary locations were the key concept introduced by Cowley and Lockwood (1992) and provide a way to understand observed flow responses to reconnection changes. We define an equilibrium boundary to be the location such that were a magnetospheric boundary (the open-closed field line boundary or the magnetopause) to be at such a location at all MLT, the flow in the magnetosphere-ionosphere system would fall to zero. The production of new open flux perturbs the boundaries from these equilibrium locations and flow results as the magnetosphere returns towards equilibrium. Note that because newly-opened flux is dragged antisunward by the solar wind, these equilibrium boundary locations will evolve with time to their final locations shown in rows 1 and 4 . Thus we need to make a distinction between the final resting place of the equilibrium boundaries (the dot-dash lines in row 1 of Fig. 3) and their locations at an intermediate instant of time (dashed lines in rows 2 and 3). In row 1, we see that the reconnection pulse has produced two effects; namely, equatorward motion of the dayside open-closed boundary and Earthward erosion of the dayside magnetopause. In Fig. 3a, a region of newly-opened flux has been appended around noon to the ionospheric polar cap (here taken to mean the region of open geomagnetic flux) and this erosion has taken place at the merging gap ( $a b$ in Fig. 1 and Fig. 3a). In Fig. 2a we see that the open flux has already moved away from the equatorial reconnection $X$-line and so the dayside equatorial magnetopause has been eroded inward in Fig. 3d; this erosion takes place at the reconnection site (AB in Fig. 1). However, the newly-open flux has not yet migrated tailward as far as $[X]_{G S M}=-30 R_{E}$ nor even to $[X]_{G S M}=0$ and thus no effect of the reconnection pulse is yet apparent in either Fig. $3 \mathrm{c}$ nor $3 \mathrm{~b}$. The final equilibrium locations of the magnetopause, shown as dot-dash lines in row 1, reflect the fact that the reconnection pulse has eroded the total flux in the dayside magnetosphere and the opened flux produced has flared the tail as it is dragged antisunward by the solar wind flow.

In row 2 of Fig. 3, for $t=4$ min (corresponding to Fig. 2c), the open flux produced by the pulse has reached $[X]_{G S M}=0$ and is perturbing the magnetospheric equilibrium there. In particular, the newly-opened flux is appended to the sunward end of the tail lobe. In this case, the dashed lines show the locations of the equilibrium boundary at this time. Note that the magnetopause at $[X]_{G S M}=-30 R_{E}$ has yet to be perturbed because the newly-opened flux has not propagated sufficiently far down the tail. In Fig. 3f, we see the flows that result at $[X]_{G S M}=0$ because the magnetosphere is returning toward the equilibrium configuration for the new amount of open flux that is present in the near-Earth magnetosphere; this flow is in the tail lobes and corresponds to the antisunward flow that has appeared in the polar cap ionosphere as part of the small convection vortices shown in Fig. 3e. This antisunward flow is taking place in the ionosphere after the reconnection pulse has ceased because the OCB between $a$ and $b$ is equatorward of its equilibrium location and is relaxing poleward. Flow in the ionosphere is incompressible, in the sense that the ionospheric field strength $B_{i}$ remains approximately constant, thus there are no sources and sinks and flow streamlines are closed loops. This means that the antisunward flow in the polar cap must be accompanied by return sunward flow in the auroral oval, completing the vortices. The antisunward flow on open field lines couples to the sunward flow on closed field lines through equatorward motion of the adiaroic polar cap boundary toward its new equilibrium position since no flux can cross that boundary. This sunward flow on closed field lines mirrors that seen in the equatorial magnetosphere in Fig. $3 \mathrm{~h}$ as the eroded dayside magnetopause relaxes back sunward and closed magnetic flux in the tail is squeezed sunward by the arrival of the new open flux in the tail lobes. 
The situation at $t=14 \mathrm{~min}$ is similar to that at $t=4 \mathrm{~min}$, except that the newly-opened flux has reached $[X]_{G S M}=$ $-30 R_{E}$ and is perturbing the magnetosphere there as well as at $[X]_{G S M}=0$ (as in Fig. 2e). In the ionosphere, this tailward expansion of the magnetospheric perturbation flow is mirrored in an expansion of the flow pattern away from noon. In column 1 of Fig. 3, $r$ and $s$ are the points sunward of which the ionospheric equilibrium boundary location is perturbed (on the dusk and dawn flanks, respectively). These migrate toward midnight as the newly-opened flux is appended to the tail at increasingly negative $[X]_{G S M}$.

The last row in Fig. 3 is the idealised situation where the magnetosphere has fully come into equilibrium with the new amount of open flux (corresponding to Fig. 2f). This may take more than an hour and so is unlikely to be realised in practice as another source of perturbation (either a second pulse of magnetopause reconnection or the onset of tail reconnection) is very likely to take place before this is achieved. Nevertheless, this idealised state is important as it describes what the magnetosphere-ionosphere system is tending toward at earlier times. In row 4 of Fig. 3, the solid lines are the OCB and magnetopause and the dotted line shows the initial OCB and magnetopause locations, just before the reconnection pulse (as also shown in row 1).

Note that at all times demonstrated in Fig. 3, the reconnection has ceased; all flow that is present is associated with adiaroic (non-reconnecting) boundaries moving toward their new equilibrium locations with less closed flux on the dayside and more open flux in the tail.

Figure 3 is concerned with a short pulse of magnetopause reconnection. Similar considerations can be applied to a burst of tail reconnection on a range of timescales. However, in this paper we restrict our attention to variations in the magnetopause reconnection rate. Generalisation for steady or variable tail reconnection can be added using similar principles, as discussed by Cowley and Lockwood (1992) and Lu et al. (2002), but will add to the complexity.

1.2 Observations of ionospheric convection responses to reconnection rate variations

A rise in the rate of open flux generation (the magnetopause reconnection voltage) is communicated from the magnetopause to the ionosphere by Alfvén waves which propagate along dayside field lines in 1-2 min. Such rapid responses were first detected by Nishida (1968a, b) who compared data from near-Earth IMF monitors with observations by dayside ground-based magnetometers. The key prediction of the Cowley-Lockwood flow-excitation model is that the majority of the ionospheric flow response to the reconnection pulse at a magnetopause $X$-line $A B$ is a localised pair of convection vortices, centred on the ends of the merging gap, $a$ and $b$, and that these vortices expand in MLT away from the merging gap as the magnetospheric tail at greater $[X]_{G S M}$ is perturbed by the newly-opened flux produced by the pulse (as illustrated in Fig. 3).
This expansion has been reported in various experimental studies. The antisunward propagation of a change in the ionospheric convection electric field was first reported by Lockwood et al. (1986), who used the EISCAT (European Incoherent Scatter) radar to observe the consequent increase in F-region ion temperatures following a southwardturning of the IMF, as seen just outside the bow shock by the AMPTE (Active Magnetospheric Particle Tracer Explorer) spacecraft. This heating is caused by collisions between the convecting ions and neutral thermospheric atoms whose motion cannot respond as rapidly. The convection increase was shown to have propagated eastward over the two beams of the EISCAT radar in the afternoon sector auroral oval (i.e. away from noon), a cross-correlation analysis showing that the rise was seen first in the beam closer to noon. The lag between the changes seen in the two beams gave a propagation speed of $2.6 \mathrm{~km} \mathrm{~s}^{-1}$ in the eastward direction around the afternoon sector, i.e. away from noon. The same technique was used to make direct observations of similar propagation of convection enhancements associated with transient cusp/cleft auroral events thought to be caused by short-lived (minute-scale) pulses of magnetopause reconnection (Lockwood et al., 1993a).

This propagation has also been found in surveys of the response time of the convection change as a function of position. The response time was found to increase with distance from a location near noon, using EISCAT radar observations of the flow in combination with AMPTE observations of the IMF close to the subsolar point of the bow shock. Etemadi et al. (1988) employed a statistical correlation analysis, while Todd et al. (1988) made a survey of event studies. A similar result was found by Saunders et al. (1992) by comparing oscillations in the $B_{z}$ component of the IMF to those seen at different stations of the CANOPUS (Canadian Auroral Network for the Open Program Unified Study) magnetometer network and, more recently, by Cowley et al. (1998), Khan and Cowley (1999) and McWilliams et al. (2001). These authors have used a variety of radar techniques to observe the expanding flow response: Etemadi et al. used vector flows generated by the EISCAT incoherent scatter radar using the beam-swinging technique (which has assumptions and limitations, cf. Freeman et al., 1991); Todd et al. used measurements of line-of-sight flow component and Cowley et al. and Khan and Cowley employed tristatic EISCAT flow observations. McWilliams et al. used data from the SuperDARN (Super-Dual Auroral Radar Network) HF coherent scatter radars with two different techniques: bistatic measurements were possible in localised regions and a more global view was obtained using the mapped potential procedure described by Ruohoniemi and Baker (1998).

The expansion speed inferred from these studies is initially of order $10 \mathrm{~km} \mathrm{~s}^{-1}$ but falls as the pattern expands such that it takes about $10 \mathrm{~min}$ to cover the entire polar ionosphere. Lockwood and Cowley 1991) pointed out that the response to a northward turning, as deduced by Knipp et al. (1991) from global radar and magnetometer data using the AMIE (Assimilative Mapping of Ionospheric Electrodynamics) method, 
also showed this expansion from the dayside to the nightside. There is some debate about the expansion rate of the peak response. The initial studies of Etemadi et al. (1988) and Todd et al. (1988) deduced that expansion took of order 10-15 min, while Kahn and Cowley (1999) found values closer to 5-10 $\mathrm{min}$, the latter also being consistent with the global MHD simulations of Slinker et al. (2000). Using magnetometer data, Murr and Hughes (2001) have demonstrated the expansion of the convection pattern but noted that the expansion velocity depended on which feature of the response was studied.

On the other hand, Ridley et al. $(1997,1998)$ have studied several cases of IMF southward turnings, as observed by the Wind satellite at locations far more distant from both the bow shock and the Sun-Earth line than the position of the AMPTE satellites in the studies discussed in the previous section. As a result, these authors needed to consider carefully the much greater uncertainties in the propagation delay from the satellite to the magnetopause. These authors inferred the ionospheric flows, using the AMIE (Assimilative Mapping of Ionosheric Electrodynamics) technique, from the magnetic deflections caused by the associated currents and detected by a network of ground-based magnetometers. The net flow was regarded as a superposition of a new pattern and the pre-existing pattern and thus the latter was subtracted from the observed flow to deduce the disturbance to the convection pattern caused by the southward turning of the IMF. Ridley et al. (1997, 1998) interpreted the results as showing a global enhancement of the flow, with no evolution of the pattern of flow; this is therefore significantly different from the conclusions of the studies discussed above which detect an expansion of the pattern. Lockwood and Cowley (1999) argue that the convection pattern expansion predicted by the Cowley-Lockwood model is, in fact, present in the case presented by Ridley et al.

Ruohoniemi and Greenwald (1998) have reported SuperDARN (Dual Auroral Radar Network) HF radar measurements of the dusk cell which they interpret as showing a nearinstantaneous global change of the convection pattern from a low-flow, northward IMF situation to a southward vigorous southward-IMF pattern. However, this interpretation is complicated by the fact that only dusk cell data are available in this case and this asymmetry opens up possible effects due an observed polarity reversal of the IMF $B_{y}$ component. In addition, the interpretation of Ruohoniemi and Greenwald does not explain the disappearance of a lobe circulation cell, characteristic of northward IMF, roughly $8 \mathrm{~min}$. prior to what they consider to be the first effects of the southward turning of the IMF. The predicted propagation lag is consistent with an interpretation in which the IMF $B_{z}$ change is responsible for the disappearance of the lobe cell and the $B_{y}$ change is responsible for the convection enhancement in the dusk cell.

\subsection{Scope of the present paper}

Resolution of the different views of the response of ionospheric convection to the onset of magnetopause reconnec- tion, as discussed in Sect. 1.2, was one of the driving forces behind the construction of a numerical model that embodies all the concepts presented in Sect. 1.1. In the present paper, we describe the numerical model and present some simulated flow patterns that are predicted in response to one example of a specified input variation of the magnetopause reconnection rate. In later papers, we will investigate the model predictions in relation to the debate about expansion of the pattern, outlined in Sect. 1.2. We will also use the model to study the various methods that have been used to derive the speed of the pattern expansion from experimental data.

\section{The model}

The model presented here is based on the concepts introduced by Cowley and Lockwood (1992). The implementation of these concepts is best discussed using Fig. 4 which shows some output data produced by the model. In Fig. 4a, the solid line shows the latitude of the $\mathrm{OCB}, \Lambda_{\mathrm{OCB}}$, as a function of MLT; the dot-dashed line shows the latitude of the equilibrium boundary location, $\Lambda_{E}$. Both of these are for a certain time (in this case $t_{s}=120 \mathrm{~s}$ into the simulation). Because the equilibrium boundary is for the amount of open flux present at that time, the total magnetic flux poleward of these two boundaries must, by definition, be equal at all times: because the ionospheric field is approximately constant at $B_{i}=5 \times 10^{-5} \mathrm{~T}$, this means that the area poleward of the two boundaries must always be the same. Once the locations of these two boundaries is known as a function of MLT, we can evaluate the velocity of poleward motion of the OCB which, in the absence of any reconnection, is taken always to be relaxing towards the equilibrium boundary with a characteristic time constant of $\tau_{\mathrm{OCB}}$. In general, we could take $\tau_{\mathrm{OCB}}$ to be a specified function of MLT, however, in this paper, we use as simpler formulation with $\tau_{\mathrm{OCB}}$ set at a constant value of $10 \mathrm{~min}$ at all MLT. This value is taken from analysis of the EISCAT-AMPTE data discussed in Sect. 1.2. Taking the time derivative yields the poleward convection velocity caused by the boundary motion:

$$
\begin{gathered}
V_{c n}\left(\mathrm{MLT}, t_{s}\right)=p \times\left[\Lambda_{E}\left(\mathrm{MLT}, t_{s}\right)-\right. \\
\left.\Lambda_{\mathrm{OCB}}\left(\mathrm{MLT}, t_{s}\right)\right] / \tau_{\mathrm{OCB}}(\mathrm{MLT}),
\end{gathered}
$$

where $p$ is the distance in the poleward direction corresponding to a unit difference in invariant latitude (here we use the value for a height $h$ of $300 \mathrm{~km}$ and thus $p$ is equal to $\left(R_{E}+h\right) \times 2 \pi / 360=116 \mathrm{~km}$ for $\Lambda_{E}$ and $\Lambda_{\mathrm{OCB}}$ in degrees). This convection velocity will, in general, differ from the poleward boundary velocity $V_{b n}$ because reconnection may be present:

$$
V_{b n}\left(\mathrm{MLT}, t_{s}\right)=V_{c n}\left(\mathrm{MLT}, t_{s}\right)-V^{\prime}\left(\mathrm{MLT}, t_{s}\right),
$$

where $V^{\prime}$ is the convection velocity, poleward across the OCB in the OCB rest frame. Thus $V^{\prime}$ is zero at the MLT of all adiaroic segments of the open closed boundary, $V^{\prime}>0$ 

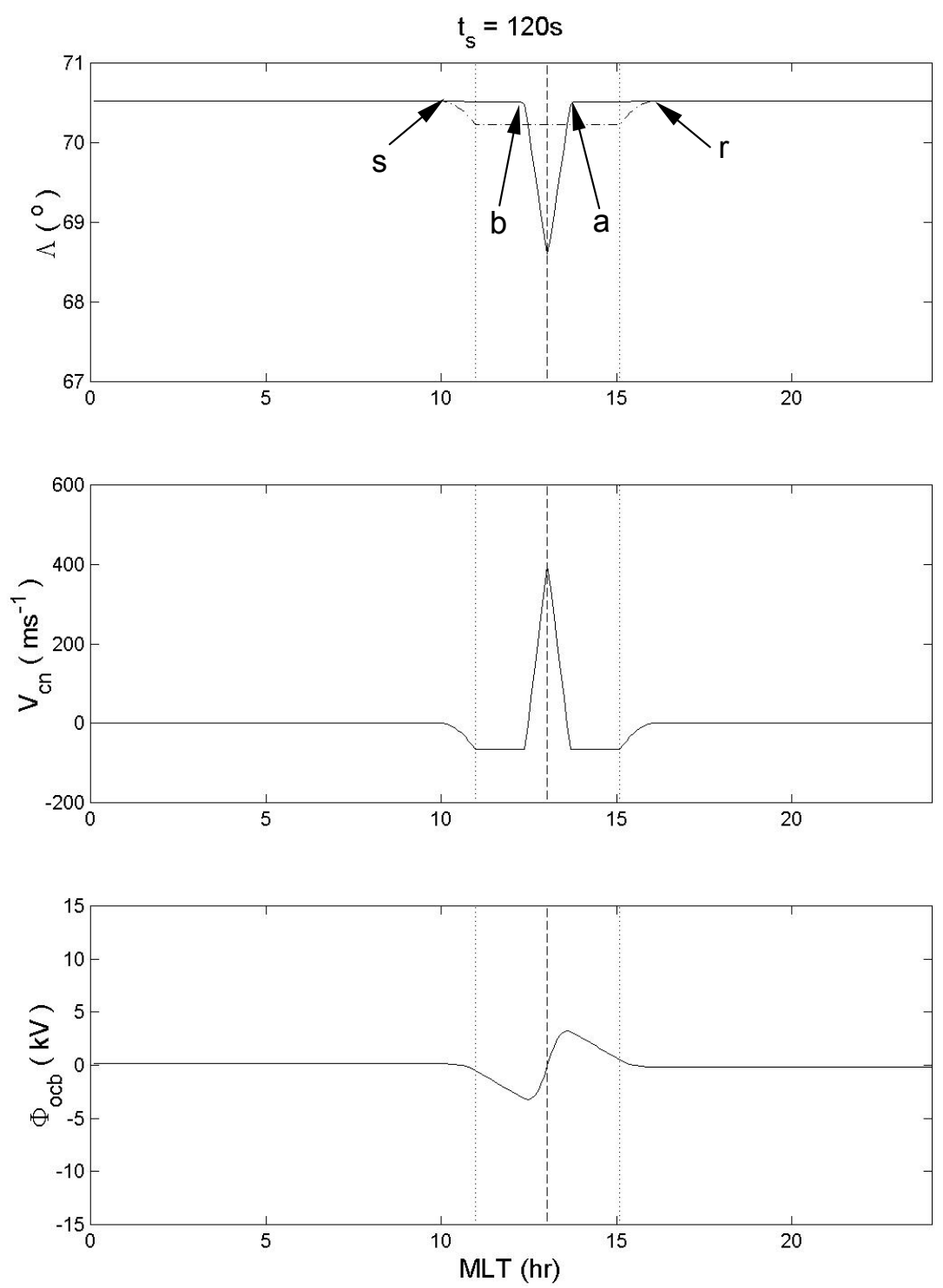

Fig. 4. Output model data for simulation time $t_{s}=120 \mathrm{~s}$ and as a function of MLT. (a) The latitude of the (solid line) open-closed field line boundary $\left(\Lambda_{\mathrm{OCB}}\right)$ and (dot-dashed line) the equilibrium boundary location $\left(\Lambda_{E}\right)$. (b) The poleward convection velocity at the OCB, $V_{c n}$. (c) The electrostatic potential around the open-closed boundary $\Phi_{\mathrm{OCB}}$. In each panel, the vertical dashed line is the centre of the merging gap and the vertical dotted lines mark the ends of the merging gap ( $a$ and $b$ ) for its maximum extent.

for a merging gap which maps to a magnetopause reconnection site where open flux is generated and $V^{\prime}<0$ for all MLT which map to a tail reconnection site. To allow for the fact that a merging gap, corresponding to a reconnection $X$-line of fixed MLT extent becomes slightly longer when the boundary erodes equatorward, a small correction is made

$$
\begin{aligned}
& V^{\prime}\left(\mathrm{MLT}, t_{s}\right)= \\
& \quad V_{o}^{\prime}\left(\mathrm{MLT}, t_{s}\right) \times \cos \left[\Lambda_{\mathrm{OCB}}\left(\mathrm{MLT}, t_{s}\right)\right] / \cos \left[\Lambda_{i}\right],
\end{aligned}
$$

where $\Lambda_{i}$ is a reference boundary invariant latitude. We use the value of $\Lambda_{\mathrm{OCB}}$ at the start of the simulation and adopt a polar cap which is initially circular and thus $\Lambda_{\mathrm{OCB}}(M L T, 0)=\Lambda_{i}$. As will be discussed in the Sect. 3, the variation of $V_{o}^{\prime}$ is specified as a function of MLT and simulation time $t_{s}$ by the tail and magnetopause reconnection variations which are inputs to the model. Thus application of Eqs. (3), (4) and (5) allows us to evaluate both the latitudinal convection velocity (positive poleward) $V_{c n}$, and the boundary motion velocity (again positive poleward) $V_{b n}$, both as a function of MLT and time $t_{s}$.

The boundary velocity $V_{b n}$ is used to update the polar cap boundary location at each MLT for the next time step of the simulation $\Delta t_{s}$ later:

$$
\begin{aligned}
& \Lambda_{\mathrm{OCB}}\left(\mathrm{MLT}, t_{s}+\Delta t_{s}\right) \\
& \quad=\Lambda_{\mathrm{OCB}}\left(\mathrm{MLT}, t_{s}\right)+\Delta t_{s} \times V_{b n} / p .
\end{aligned}
$$


The velocity $V_{c n}$ corresponding to the boundary locations shown in Fig. 4a is presented as a function of MLT in Fig. 4b: because $V_{c n}=E_{T} / B$, this specifies the distribution of electric field tangential to the boundary, $E_{T}$, and the ionospheric potential around the polar cap boundary, $\Phi_{\mathrm{OCB}}$, by integrating with respect to the angle $\varphi$ (which corresponds to MLT) we derive the potential as a function of MLT for the time $t_{s}$

$$
\begin{aligned}
& \Phi_{\mathrm{OCB}}\left(\operatorname{MLT}, t_{s}\right)= \\
& \quad \int_{0}^{\varphi} V_{c n}\left(\mathrm{MLT}, t_{s}\right) B_{i}\left(R_{E}+h\right) \cos \left[\Lambda_{\mathrm{OCB}\left(\mathrm{MLT}, t_{s}\right)}\right) d \varphi .
\end{aligned}
$$

We define the angle $\varphi$ to be positive toward dusk (see Fig. 3a) and to be the angle subtended at the centre of the polar cap by the centre of the reconnection merging gap and the MLT meridian in question. The distribution of potential around the OCB shown in Fig. 4c is typical of observations ( $\mathrm{Lu}$ et al., 1989) and is used to find the potential distribution throughout the polar cap and auroral oval by assuming ionospheric conductivity is independent of position and solving Laplace's equation. We simplify this calculation here with the approximation that the OCB is circular (at $\Lambda=\Lambda_{o}$ ) as this allows the equations given by Freeman et al. (1991) to be applied (see their Appendix A). After taking $F$, the Fourier transform of $\left.\Phi_{\mathrm{OCB}}\left(\mathrm{MLT}, t_{s}\right)\right)$ sampled using $N$ points $(24 / N) \mathrm{h}$ apart in MLT, we evaluate the coefficients $a_{m}$ and $b_{m}$ from:

$$
\begin{gathered}
m>0, \quad a_{m}=-2 I\{F(m+1)\} / N \\
m=0, \quad a_{m}=0 \\
m>0, \quad b_{m}=2 R\{F(m+1)\} / N, \\
m=0, \quad b_{m}=F(1) / N,
\end{gathered}
$$

where $R\{F\}$ and $I\{F\}$ are the real and imaginary parts of $F$. To allow us to use a fast digital Fourier transform here, we adopt $N=256$. The solution to Laplace's equation is facilitated by replacing latitude by a parameter $x$ :

$x=\log _{e}\{\tan ([(\pi / 2)-\Lambda] / 2)\}$,

which has a value $x_{o}$ at the circular convection boundary at $\Lambda=\Lambda_{o}$. Different equations apply to the convection polar cap $\left(\Lambda \geq \Lambda_{o}\right)$ and the convection auroral oval $\left(\Lambda \leq \Lambda_{o}\right)$. The value of $\Lambda_{o}$ used is the latitude at the ends of the merging gap (the points $a$ and $b$ in Fig. 3). Outside of the bulge on the OCB between $a$ and $b$, the actual OCB in the simulation presented here is rarely more than $1^{\circ}$ (corresponding to $p=116 \mathrm{~km}$ ) from this idealised circular boundary and the error introduced by using a circular convection boundary is small. Inside the polar cap bulge, caused by erosion between $a$ and $b$, the flow is taken to be poleward at speed $V_{c n}$ and equatorward of the bulge region, the sunward flow streamlines are shifted equatorward by the thickness of the bulge at that MLT. This ensures that streamlines are continuous across the OCB.
Inside the convection polar cap $\Lambda \geq \Lambda_{o}$, the potential $\Phi$ is determined from:

$$
\begin{aligned}
& d \Phi / d x=\Sigma_{m} m e^{m(x-x o)}\left[a_{m} \sin (m \varphi)+b_{m} \cos (m \varphi)\right], \\
& d \Phi / d \varphi=\Sigma_{m} m e^{m(x-x o)}\left[a_{m} \cos (m \varphi)-b_{m} \sin (m \varphi)\right]
\end{aligned}
$$

and the northward and eastward flow velocities, respectively $V_{N}$ and $V_{E}$ from

$$
\begin{aligned}
& V_{N}=-\{d \Phi / d \varphi\} /\left\{B_{i}\left(R_{E}+h\right) \sin [(\pi / 2)-\Lambda]\right\} \\
& V_{E}=-\{d \Phi / d x\} /\left\{B_{i}\left(R_{E}+h\right) \sin [(\pi / 2)-\Lambda]\right\} .
\end{aligned}
$$

In the convection auroral oval $\left(\Lambda \geq \Lambda \geq \Lambda_{1}\right)$ the convection pattern is restricted to poleward of a latitude $\Lambda=\Lambda_{1}$ which, by Eq. (10), corresponds to $x=x_{1}$. In the present simulation, a fixed value of $\Lambda_{1}=65^{\circ}$ is employed: this is the latitude of the region 2 ring of field-aligned current. To accommodate the bulge in the OCB at the merging gap, a new parameter $x^{\prime}(\varphi)$ is used. Outside the merging gap $\left(\varphi_{a}<\varphi<\varphi_{b}\right)$, $x^{\prime}(\varphi)$ is the same as $x$, as given by Eq. (10). Inside the merging gap $\left(\varphi<\varphi_{a}\right.$ and $\left.\varphi>\varphi_{b}\right)$ we introduce a latitudinal shift equal to the latitudinal separation of the OCB and the convection boundary at that MLT, giving a modified latitude, $\Lambda^{\prime}(\varphi)=\Lambda+\left\{\Lambda_{o}(\varphi)-\Lambda_{\mathrm{OCB}}(\varphi)\right\}$ :

$$
\begin{aligned}
& \varphi_{a}<\varphi<\varphi_{b}: x^{\prime}(\varphi)=x \\
& \varphi<\varphi_{a} \text { and } \varphi>\varphi_{b}: \\
& x^{\prime}(\varphi)=\log _{e} \tan \left(\left[(\pi / 2)-\Lambda^{\prime}(\varphi)\right] / 2\right) \\
& \left.d \Phi / d x^{\prime}=\Sigma_{m} m\left[\cosh \left\{m\left(x^{\prime}-x_{1}\right)\right\}\right] / \sinh m\left(x_{o}-x_{1}\right)\right] \times \\
& \quad\left[a_{m} \sin (m \varphi)+b_{m} \cos (m \varphi)\right] \\
& d \Phi / d \varphi=\Sigma_{m} m\left[\sinh \left\{m\left(x^{\prime}-x_{1}\right)\right\} / \sinh \left\{m\left(x_{o}-x_{1}\right)\right\}\right] \times \\
& \quad\left[a_{m} \cos (m \varphi)-b_{m} \sin (m \varphi)\right] .
\end{aligned}
$$

The northward and eastward flow velocities, respectively $V_{N}$ and $V_{E}$, are derived from

$$
\begin{aligned}
\Lambda< & \Lambda_{\mathrm{OCB}} \quad \& \quad \Lambda<\Lambda_{o}: \\
& V_{N}=-\{d \Phi / d \varphi\} /\left\{B_{i}\left(R_{E}+h\right) \sin \left[(\pi / 2)-\Lambda^{\prime}\right]\right\} \\
& \Lambda_{o}>\Lambda \geq \Lambda_{\mathrm{OCB}}: \quad V_{N}=V_{c n} . \\
\Lambda< & \Lambda_{\mathrm{OCB}} \quad \& \quad \Lambda<\Lambda_{o}: \\
& V_{E}=-\{d \Phi / d x\} /\left\{B_{i}\left(R_{E}+h\right) \sin \left[(\pi / 2)-\Lambda^{\prime}\right]\right\} \\
& \Lambda_{o}>\Lambda \geq \Lambda_{\mathrm{OCB}}: \quad V_{E}=0 .
\end{aligned}
$$

The condition $\Lambda_{o}>\Lambda \geq \Lambda_{\mathrm{OCB}}$ only applies in the bulge of newly-opened flux equatorward of the merging gap ab, where the flow is taken to be uniformly poleward (i.e. with the convection velocity at the merging gap). 
Thus the model can generate the full convection pattern once the variation of the actual and equilibrium boundary latitudes with MLT has been evaluated. To do this, we use the concepts described schematically in Fig. 3 and the points $r$ and $s$, sunward of which the equilibrium boundary is perturbed equatorward by the reconnection pulse, which migrates towards midnight; this motion reflects the tailward evolution of the newly-opened flux produced by the reconnection pulse. For example, the points $r$ and $s$ are at $\varphi=\varphi_{r}$ and $\varphi=\varphi_{s}$ and are initially placed at $a$ and $b$ at the start of the reconnection pulse, moving longitudinally at angular velocities $d \varphi_{r} / d t_{s}$ and $d \varphi_{s} / d t_{s}=-d \varphi_{r} / d t_{s}$, respectively, (such that $r$ and $s$ both travel at the same speed toward the nightside, around the dusk and dawn flanks, respectively). This angular speed is an input to the model. Although $d \varphi_{r} / d t_{s}$ could, in general, be a function of MLT, we here adopt a constant value of $0.25^{\circ} \mathrm{s}^{-1}$ (corresponding to $1 \mathrm{~h}$ of MLT per min). Thus the expansion takes $12 \mathrm{~min}$ to propagate to all MLT. The rate of equatorward motion of the equilibrium boundary at a given MLT is controlled by the reconnection rate history. From this we define a propagation time, $\Delta t_{\varphi}$, from the ends of the merging gap $a$ and $b$ for each MLT. Outside the merging gap on the duskside, $\pi>\varphi>\varphi_{a}$

$\Delta t_{\varphi}=\left(\varphi-\varphi_{a}\right) /\left|d \varphi_{r} / d t_{s}\right|$.

Outside the merging gap on the dawnside, $\pi<\varphi<\varphi_{b}$

$\Delta t_{\varphi}=\left(\varphi_{b}-\varphi\right) /\left|d \varphi_{r} / d t_{s}\right|$.

Inside the merging gap $\varphi<\varphi_{a}$ and $\varphi>\varphi_{b}$

$\Delta t_{\varphi}=0$.

Using this propagation delay we can update the equilibrium boundary latitude at each MLT using the reconnection rate history:

$$
\begin{array}{r}
\Lambda_{E}\left(\mathrm{MLT}, t_{s}+\Delta t_{s}\right)=\Lambda_{E}\left(M L T, t_{s}\right)+ \\
k\left(t_{s}\right) \times \Delta t_{s} \times V_{o}^{\prime}\left(\varphi=0, t_{s}-\Delta t_{\varphi}\right),
\end{array}
$$

where $V_{o}^{\prime}\left(\varphi=0, t_{s}-\Delta t_{\varphi}\right)$ is the ionospheric velocity corresponding to the reconnection rate at the centre of the merging gap at the time $\Delta t_{\varphi}$ earlier. The normalising constant $k$ is evaluated at each time $t_{s}$ to ensure that the total magnetic flux poleward of the equilibrium boundary is always the same as the polar cap open flux $F_{p c}$ (poleward of the OCB) at the same time.

The polar cap flux $F_{p c}$ is updated using:

$$
\begin{aligned}
& F_{p c}\left(t_{s}+\Delta t_{s}\right)=F_{p c}\left(t_{s}\right)+ \\
& \Delta t_{s} B_{i}\left(R_{E}+h\right) \cos \left(\Lambda_{o}\right) \int_{0}^{2 \pi} V_{o}^{\prime}(\varphi) d \varphi .
\end{aligned}
$$

This scheme for evolving the OCB and equilibrium boundaries is demonstrated by comparing Figs. 4 and 5. Figure 5 is the same as Fig. 4, but for a later time of $t_{s}=360 \mathrm{~s}$. It can be seen, in Fig. 5a, that the points $r$ and $s$ have evolved towards the nightside and that the erosion of the OCB towards lower latitudes has continued in the merging gap $a b$ which has expanded in length. Outside the merging gap, between $s$ and $b$ and between $a$ and $r$, the evolution of the OCB towards the equilibrium boundary can be seen to have begun. Figures $5 b$ and $\mathrm{c}$ show that the flow speeds and convection voltage have also increased.

In implementing the model presented here, we only consider changes and boundaries after information about them has arrived in the ionosphere; this removes the need to specify the Alfvén wave propagation time, $t_{A}$, along the field lines from the reconnection site. (Therefore, in applications where the reconnection rate is specified using input IMF data from an upstream interplanetary monitor, this propagation time, $t_{A}$, should be added to the satellite-to-magnetopause travel time). In the simulation presented here, the flow across the merging gap, $V_{o}^{\prime}$, begins to increase at $t_{s}=60 \mathrm{~s}$ (see Sect. 3) and thus the magnetopause reconnection commenced at $t_{s}=60-t_{A}$. To be consistent, we have to define the polar cap flux Fpc to be the open flux of which the ionosphere has "knowledge"-which was the open flux threading the magnetopause at a time $t_{A}$ earlier.

Note that the model does not require us to define where open field lines are generated on the magnetopause - other than the MLT of the reconnection $X$-line footprints in the ionosphere must be specified, which is done by the input reconnection rate variation specification, $V_{o}^{\prime}\left(\varphi, t_{s}\right)$. Thus there is no assumption of either component or anti-parallel reconnection. In principle, there may be additional effects relating to the time it takes for newly-opened flux to evolve into the tail from different reconnection sites; however, proper evaluation would also require specification of the sheath flow over the whole of the reconnecting magnetopause which requires global MHD modelling. These effects would be a perturbation to the simple concept of the expansion of the equilibrium boundary perturbation used here, away from noon at an angular speed $d \varphi_{r} / d t_{s}$.

The model contains a number of simplifications and assumptions which have been discussed in this section as they arose. Table 1 collects these assumptions together and gives a brief analysis of the implications and limitations that each sets and any mitigating steps that can be used to reduce the uncertainties they cause.

\section{An example input reconnection scenario}

In order to run the model, the reconnection rate behaviour in space and time must be specified as an input. This is done by prescribing the ionospheric flow velocity $V^{\prime}\left(\mathrm{MLT}, t_{s}\right)$ across the OCB in its own rest frame. To remove complications caused by the length of the merging gap $a b$ changing with latitudinal motions, we specify the corresponding velocity $V^{\prime}\left(\mathrm{MLT}, t_{s}\right)$ for a hypothetical boundary at constant latitude $\Lambda_{o}$ and then use Eq. (5).

In the discussion of the physical basis of the model, the reconnection rate variation (in space and time) that adds up to a pulse of magnetic flux transfer across the merging gap 
Table 1. Summary of assumptions and approximations

\begin{tabular}{|c|c|}
\hline Assumption or Approximation & Implications, Limitations, Mitigation \\
\hline The ionospheric conductivity is spatially uniform & $\begin{array}{l}\text { Model will not reproduce the tendency for flows to avoid regions of high } \\
\text { conductivity in time-dependent cases. Flow in regions of strong, }>1 \mathrm{keV} \\
\text { precipitation (e.g. substorm expansions) will not be well reproduced. }\end{array}$ \\
\hline $\begin{array}{l}\text { The equilibrium boundary perturbation spreads from } \\
\text { noon at a constant angular speed of }\left|d \varphi_{r} / d t_{S}\right| \text { on both } \\
\text { dawn and dusk flank }\end{array}$ & $\begin{array}{l}\text { Second-order corrections for the reconnection location, sheath flow pattern } \\
\text { and mapping of field lines from the tail flank to the polar cap boundary } \\
\text { could, in principle, be made but would require detailed knowledge of each } \\
\text { factor. }\end{array}$ \\
\hline$\left|d \varphi_{r} / d t_{s}\right|=0.25^{\circ} \mathrm{s}^{-1}$ & $\begin{array}{l}d \varphi_{r} / d t_{s} \text { should increase with increased solar wind speed } V_{s w} \text {. The effect } \\
\text { of various values of } d \varphi_{r} / d t_{S} \text { will be investigated by a parametric study and } \\
\text { by case studies to find the best-fit to observed flows using upstream IMF } \\
\text { data. }\end{array}$ \\
\hline $\begin{array}{l}\text { The time constant for returning boundaries to their equi- } \\
\text { librium locations, } \tau_{\mathrm{OCB}} \text { is the same at all MLT. }\end{array}$ & $\begin{array}{l}\text { Allowance could readily be made for } \tau_{\mathrm{OCB}}(M L T) \text { if it were better specified } \\
\text { by observations. }\end{array}$ \\
\hline$\tau_{\mathrm{OCB}}=10 \min$ & $\begin{array}{l}\text { The effect of various values of } \tau_{\mathrm{OCB}} \text { will be investigated by a parametric } \\
\text { study and by case studies to find the best-fit to observed flows using up- } \\
\text { stream IMF data. }\end{array}$ \\
\hline $\begin{array}{l}\text { Maximum dayside merging gap extent is } 11: 00- \\
\text { 15:00 } \operatorname{MLT}\left(\varphi_{a}=30^{\circ} ; \varphi_{b}=330^{\circ}\right)\end{array}$ & $\begin{array}{l}\text { Can be matched to data using flow and magnetometer data and/or using } \\
\text { global images of the proton aurora. }\end{array}$ \\
\hline $\begin{array}{l}\text { Region } 2 \text { current shielding confines convection to pole- } \\
\text { ward of } \Lambda_{1}=65^{\circ}\end{array}$ & Smaller $\Lambda_{1}$ could be used to model more active conditions. \\
\hline Initial polar cap flux, $F_{p c}=8 \times 10^{8} \mathrm{~Wb}$ & $\begin{array}{l}\text { Model could be run using input IMF data for long enough for initial condi- } \\
\text { tions to no longer have an effect. Or, initial } F_{p c} \text { could be chosen to match } \\
\Lambda_{i} \text { to observations of OCB. }\end{array}$ \\
\hline Departures from a circular polar cap are small. & $\begin{array}{l}\text { Analytic solution of Laplace's equation not available for irregular polar cap } \\
\text { shapes. Convection boundary is shifted slightly equatorward of OCB on the } \\
\text { nightside. }\end{array}$ \\
\hline $\operatorname{IMF} B_{y} \approx 0$ & $\begin{array}{l}\text { No simple way of introducing the cusp field-aligned currents and the } \\
\text { Svalgaard-Mansurov effect. Flows on newly opened flux will not have the } \\
\text { have longitudinal component observed when }\left|B_{y}\right| \gg 0 \text {. }\end{array}$ \\
\hline $\begin{array}{l}\text { Reconnection rate variation in space and time is input } \\
\text { to the model via } V_{o}^{\prime}\left(M L T, t_{S}\right)\end{array}$ & $\begin{array}{l}V_{o}^{\prime}\left(M L T, t_{S}\right) \text { can contain expansion along the } X \text {-line. Reconnection rate at } \\
\text { each MLT can show either a model variation or a variation set by the IMF } \\
\text { observed by an upstream monitor. }\end{array}$ \\
\hline
\end{tabular}

$a b$ was considered in general terms and not specified. In the simulation presented here, the reconnection takes place at a single magnetopause $X$-line $A B$, the merging gap of which $a b$ is centred at 13:00 MLT (corresponding to $\varphi=0$ ) and which has a maximum extent of 11:00-15:00 MLT (i.e. $a$ is at 15:00 MLT and so $\varphi_{a}=30^{\circ} ; b$ is at 11:00 MLT and $\left.\varphi_{b}=330^{\circ}\right)$. The simulation commences with a pre-existing open flux of $F_{P C}=8 \times 10^{8} \mathrm{~Wb}$. Each reconnection pulse starts at the $X$-line centre and expands towards both dusk and dawn (to $a$ and $b$, respectively) at a rate $\left(d\left|\varphi_{X}\right| / d t_{S}\right)$ which we set to $0.167^{\circ} \mathrm{s}^{-1}$, corresponding to $1 \mathrm{~h}$ of MLT per $1.5 \mathrm{~min}$. Similarly, the end of each reconnection pulse is first seen at 13:00 MLT, $\delta t=1 \mathrm{~min}$ after the onset, and propagates towards both $a$ and $b$ at the same rate. Thus each pulse causes the reconnection to be active at any MLT between $a$ and $b$ for one minute. Two such pulses are included in the present simulation, with onsets at $t_{s}=1 \mathrm{~min}$ and $t_{s}=9 \mathrm{~min}$. The background reconnection rate outside the two pulses is taken to be zero.

At the centre of the $X$-line, the reconnection rate within the pulses is such that, over the repetition period of $\tau=8 \mathrm{~min}$, the average poleward convection speed produced would be $V_{x}^{*}=500 \mathrm{~ms}$. This means, because $V_{o}^{\prime}$ is always zero outside the pulses, that $V_{o}^{\prime}=V_{x}^{*} \times(\tau / \delta t)$ within them at the centre of the $X$-line. Lastly, the amplitude of the pulses is decreased to zero as they approach $a$ and $b$. This is necessary to avoid discontinuities in the OCB forming at $a$ and $b$. We here employ a cosinusoidal decrease with distance away 

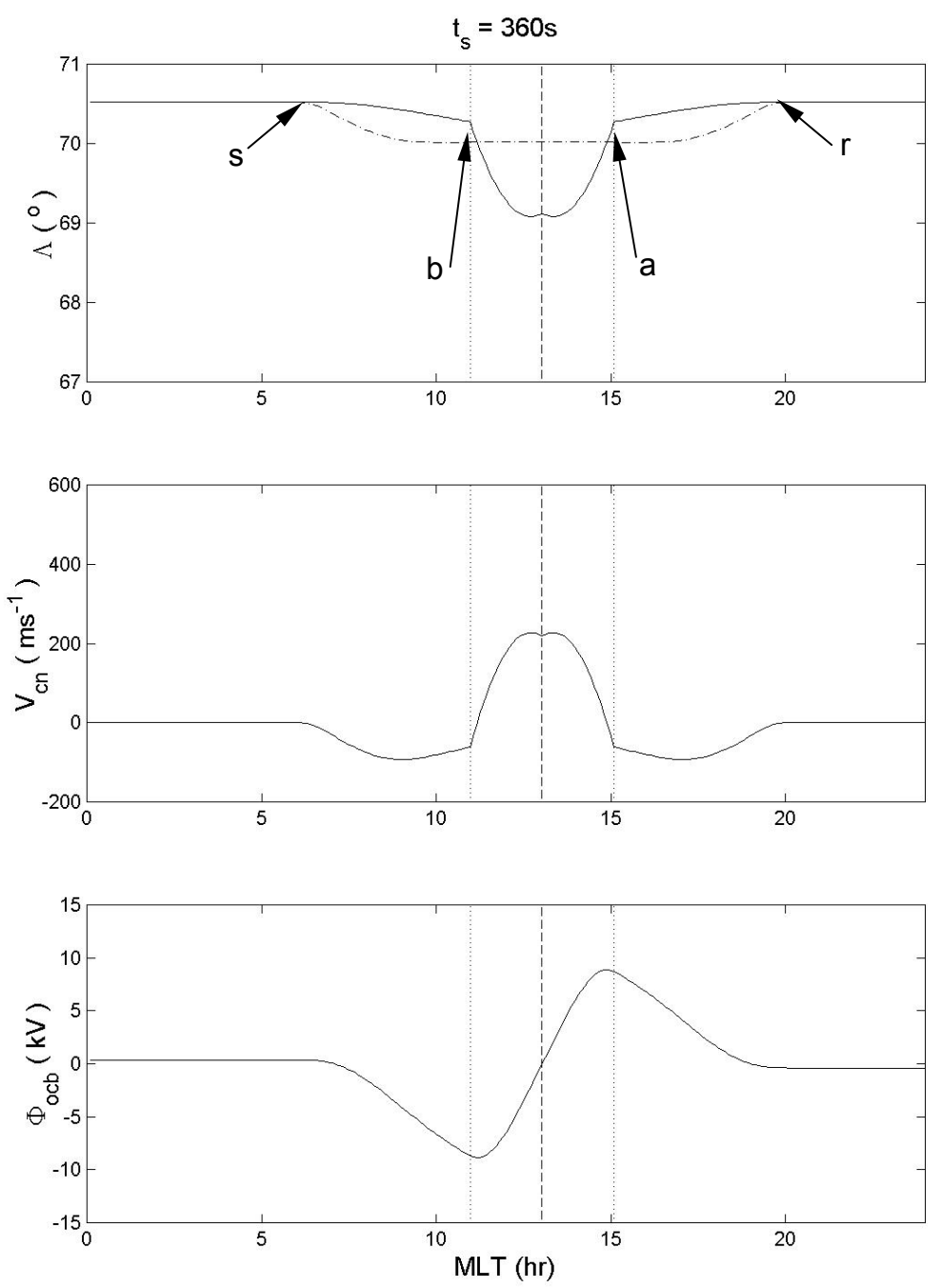

Fig. 5. Same as Fig. 4, but for simulation time $t_{s}=360 \mathrm{~s}$.

from the centre of the merging gap:

$$
\begin{gathered}
\text { for } \quad \varphi<\varphi_{a}: \quad V_{o}^{\prime}\left(\varphi, t_{s}\right)= \\
\Omega\left(\varphi, t_{s}\right) \times V_{x}^{*} \times(\tau / \delta t) \times \cos \left\{(\pi / 2) \times\left(\varphi / \varphi_{a}\right)\right\} \\
\text { for } \quad \varphi>\varphi_{b}: \quad V_{o}^{\prime}\left(\varphi, t_{s}\right)=\Omega\left(\varphi, t_{s}\right) \times V_{x}^{*} \times \\
(\tau / \delta t) \times \cos \left\{(\pi / 2) \times\left(|2 \pi-\varphi| /\left|2 \pi-\varphi_{b}\right|\right)\right\},
\end{gathered}
$$

where $\Omega\left(\varphi, t_{s}\right)$ is equal to unity for a time $t_{s}$ for which the $\varphi$ considered is inside a propagating reconnection pulse and equal to zero at all other times, as prescribed above.

This specification of the reconnection behaviour, as a function of simulation time $t_{s}$ and MLT, is the input to the convection model described in the previous section. Each pulse in this example causes the open flux to increase by $0.185 \times 10^{8} \mathrm{~Wb}$ in total. At the start of the simulation presented here, the pre-existing open flux is $F_{p c}=8.00 \times 10^{8} \mathrm{~Wb}$, rising to $8.185 \times 10^{8} \mathrm{~Wb}$ at the end of the first pulse and $8.37 \times 10^{8} \mathrm{~Wb}$ at the end of the second; thus each pulse adds $\sim 2.3 \%$ to the polar cap.

\section{Results}

Figure 6 demonstrates the presentation format of the model output. The top panel shows the variation with simulation time $t_{s}$ of two key voltages: the blue line gives the reconnection voltage $\Phi_{X L}$ produced by the input scheme discussed in the previous section, the red line shows the polar cap voltage $\Phi_{P C}$ that results from the convection model. The voltage $\Phi_{X L}$ is total instantaneous voltage that exists along the dayside ionospheric merging gap in its own frame of reference. Note that $\Phi_{X L}$ has been divided by a factor of 5 to enable display on the same scale as $\Phi_{P C}$. The vertical green line marks the $t_{s}$ of the convection pattern shown in the lower panel (the example presented in Fig. 6 is for $t_{s}=660 \mathrm{~s}$ ). Under the top panel the current values of $\Phi_{P C}$ and $\Phi_{X L}$ are given in red and blue text, respectively.

The reconnection voltage, $\Phi_{X L}$, shows the input of two pulses to this simulation. The prescribed motion of the square-wave reconnection rate pulses and the cosinusoidal decrease in the pulse amplitude gives the variation of $\Phi_{X L}$ 


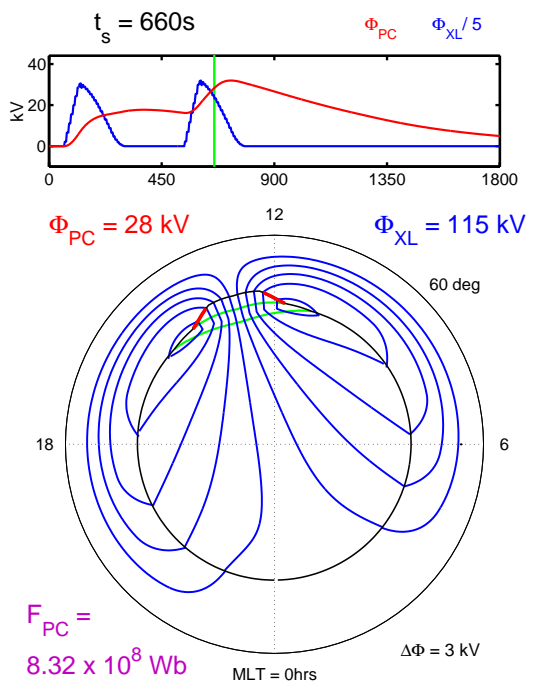

Fig. 6. An example of the output from the model. The top panel gives the variation with $t_{S}$ of $\Phi_{X L} / 5$, where $\Phi_{X L}$ is the input reconnection voltage (in blue) and of the resulting transpolar voltage $\Phi_{P C}$ (in red). The vertical green line gives the simulation time $t_{S}$ of the convection pattern shown in the MLT-invariant latitude $(\Lambda)$ plot beneath. In this lower plot, 12:00 MLT is to the top and 06:00 MLT to the right and the outer circle is $\Lambda=60^{\circ}$. Adiaroic (non-reconnecting) segments of the OCB are shown in black, reconnecting segments of the OCB in red. Equipotential contours, $\Delta \Phi=3 \mathrm{kV}$ apart, are shown in blue. The green lines delineate flux opened by the two reconnection pulses. The current values of the voltages $\Phi_{X L}$ and $\Phi_{P C}$ and of the polar cap flux $F_{p c}$ are given in blue, red and mauve text, respectively.

shown, with a steep rise after the onset of each pulse followed by a slower decay. The peak $\Phi_{X L}$ in each pulse is about $150 \mathrm{kV}$. The polar cap voltage $\Phi_{P C}$ is the difference between the maximum and minimum potential produced in the ionosphere. The red line shows that this increases during each reconnection pulse but decays slowly after the pulse. In the simulation presented here, with the pulses 8 min apart, the flow voltage due to the first pulse has already peaked near $20 \mathrm{kV}$ but only decayed fractionally from this peak when the second pulse occurs and raises $\Phi_{P C}$ to above $35 \mathrm{kV}$. At the end of the simulation, at $t_{s}=1800 \mathrm{~s}, \Phi_{P C}$ has decayed to about $5 \mathrm{kV}$.

The lower part of Fig. 6 gives, in blue, the convection streamlines at the $t_{s}$ shown. The separation of the streamlines shown is $\Delta \Phi=3 \mathrm{kV}$ in all cases shown here. In this plot, 12:00 MLT is to the top, 06:00 MLT to the right and 18:00 MLT to the left. The outer black circle gives the latitude $\Lambda=60^{\circ}$, but flow is confined to poleward of $\Lambda_{1}=65^{\circ}$ (effectively the latitude of the region $2 \mathrm{ring}$ ). The OCB is shown in black where it is adiaroic and in red where reconnection is active $\left(V^{\prime}>0\right)$. The green lines delineate the field lines opened during the two reconnection pulses. The value of the open flux $F_{P C}$ is given in the bottom left in mauve.

Several features can be noted in this example. Firstly, the convection reversal boundary lies equatorward of the OCB at all MLT outside the merging gap. This is an artefact of the model and results from the use of a simplifying circular convection boundary which gives $\Lambda_{o}<\Lambda_{\mathrm{OCB}}$ at these MLT. Two active reconnecting $\left(V^{\prime}>0\right)$ segments of the OCB can be seen at this $t_{s}$ which are propagating away from the centre of the merging gap (13:00 MLT); in this case they are part of the second of the two input reconnection pulses.

Figure 6 is one frame in a sequence of patterns which is summarised in Fig. 7. Figure $7 \mathrm{a}$ is for $t_{s}=2 \mathrm{~min}$, one min after the Alfvén wave launched by the reconnection onset reached the ionosphere: a number of features can be observed. The effects of the active reconnection segments (in red) can be seen in the equatorward erosion of the OCB that it has caused. The reconnection voltage, the total rate of flux transfer into the open field line region, is $\Phi_{X L}=171 \mathrm{kV}$ at this time, whereas the polar cap flow voltage is just $\Phi_{P C}=7 \mathrm{kV}$ (thus, just two flow streamlines can be seen in Fig. 7a in the localised vortices because a contour separation of $\Delta \Phi=3 \mathrm{kV}$ is employed). The large difference between $\Phi_{X L}$ and $\Phi_{P C}$ is because, initially, the reconnection pulse predominantly causes equatorward erosion of the OCB within the merging gap rather than poleward convection. At the very centre of the bulge of newly-opened flux, the signature of the switch-off of the reconnection can be seen in Fig. 7a, having just begun and causing the appearance of an adiaroic boundary segment at 13:00 MLT. Figure $7 \mathrm{~b}$ is for $t_{s}=3 \mathrm{~min}$ and both the signatures of onset and switch-off of the reconnection have moved away from 13:00 LT towards both dawn and dusk. This azimuthal motion of the region of active reconnection is as proposed and deduced from observations by Lockwood et al. (1993a), Lockwood (1994), Smith and Lockwood (1996), Milan et al. (2000) and McWilliams et al. (2001). The flow pattern has enhanced $\left(\Phi_{P C}=13 k V\right)$, even though the reconnection voltage has begun to decrease $\left(\Phi_{P C}=111 \mathrm{kV}\right)$. The flow pattern has expanded spatially towards both dawn and dusk. This early expansion reflects a mixture of the expansions of both the active merging gap and of the disturbance to the equilibrium boundary. The changes seen between Fig. 7a and $\mathrm{b}$ all continue until $t_{s}=9$ min when the second reconnection pulse commences. When $t_{s}=10 \mathrm{~min}$, this second pulse has produced a second equatorward erosion of the OCB, almost identical to the first. However, Figs. 7h-6j $\left(t_{s}=10-12 \mathrm{~min}\right)$ show a convection response to the second pulse that is considerably different to the first, in that the response is not localised and expanding but has elements of a global instantaneous response. The transpolar voltage peaks at $\Phi_{P C}=32 \mathrm{kV}$ at $t_{s}=12 \mathrm{~min}$, just before the reconnection voltage due to the second pulse decays to zero. Subsequently, the convection pattern decays in a shape-preserving manner with potential contours migrating towards the OCB at dawn and dusk where they disappear. This is illustrated by comparison of Figs. $7 \mathrm{j}$, $\mathrm{k}$ and 1, for $t_{s}$ of $12 \mathrm{~min}, 16 \mathrm{~min}$ and $20 \mathrm{~min}$, respectively.

Note that the boundary erosion predicted by the model, due to each pulse, is of order $1-2^{\circ}$ of latitude (see Figs. 4, 5 and 7), corresponding to $116-232 \mathrm{~km}$. This is consistent with 

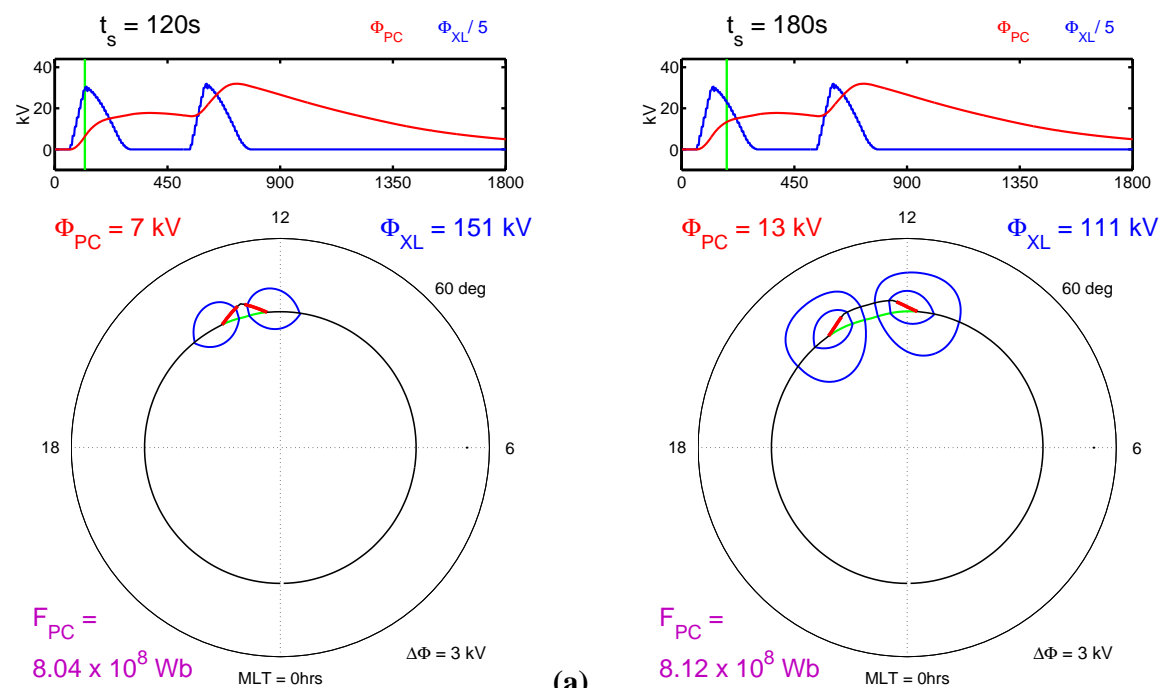

(a)

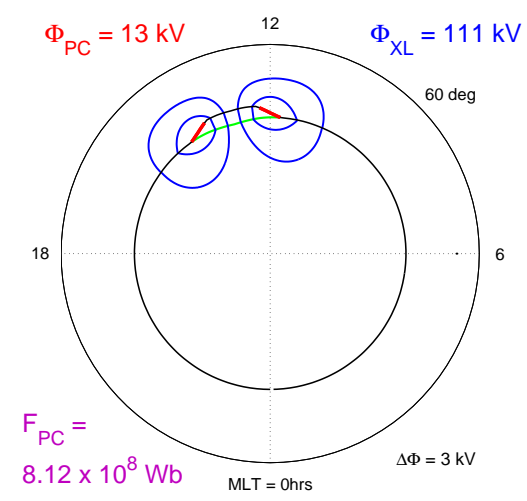

(b)
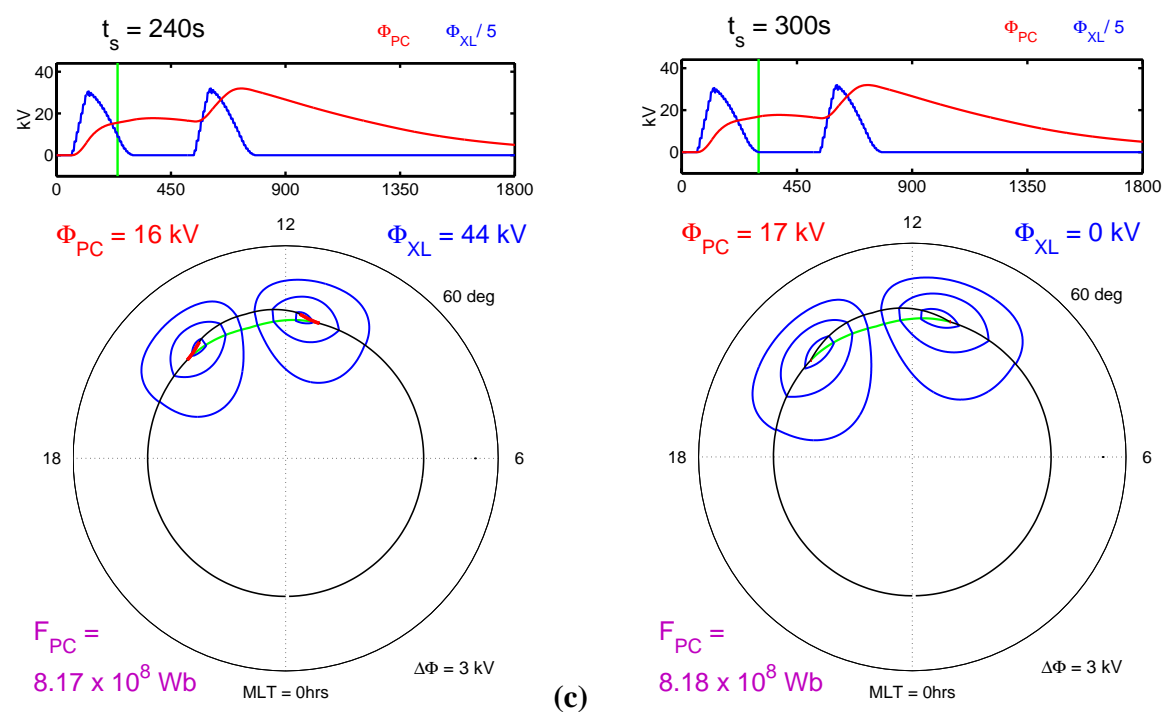

(c)
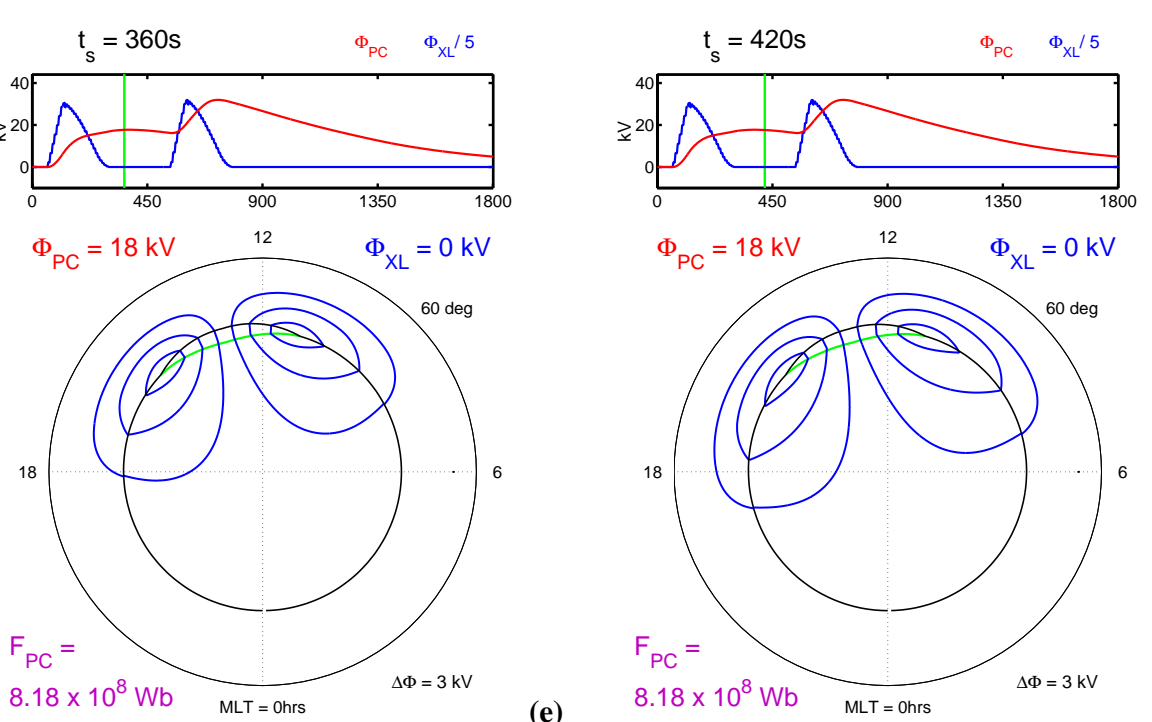

(e)

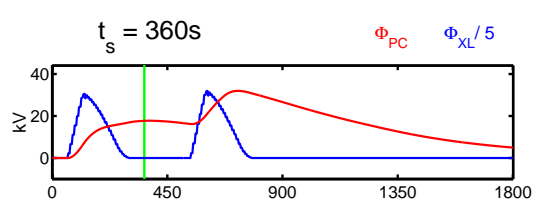

(d)

Fig. 7. Selected output convection patterns, each frame using the same format as Fig. 6 . Note that the frames are not equally spaced in simulation time, $t_{s}$. 

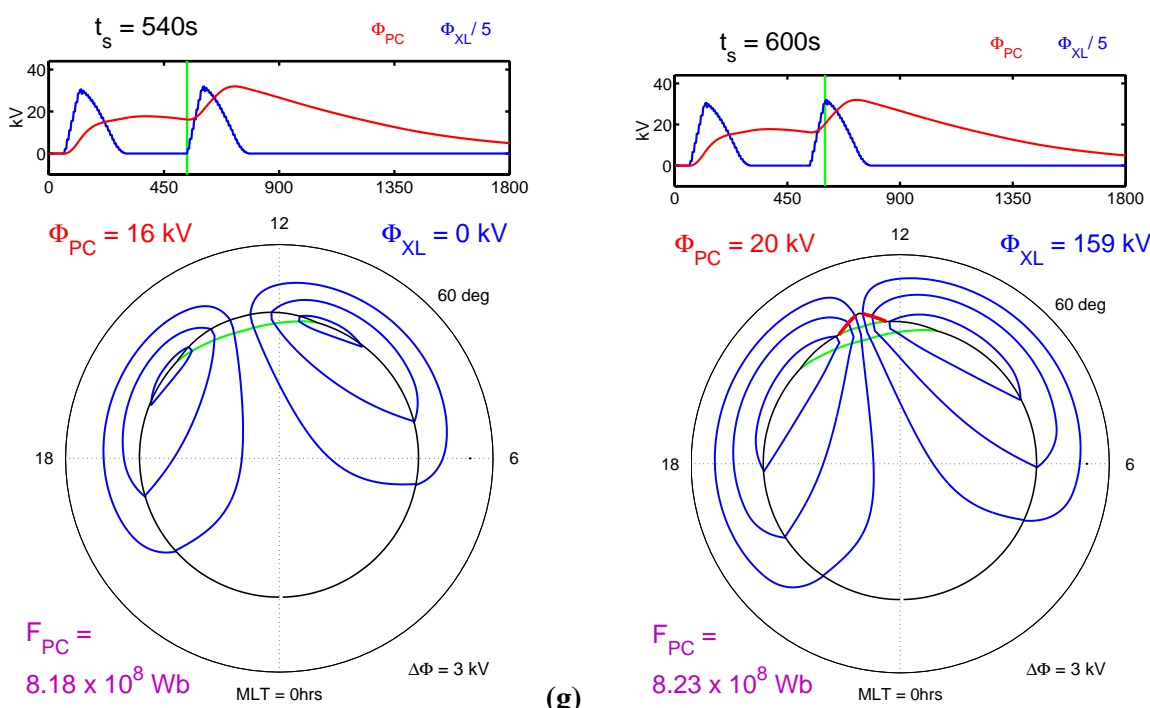

(g)
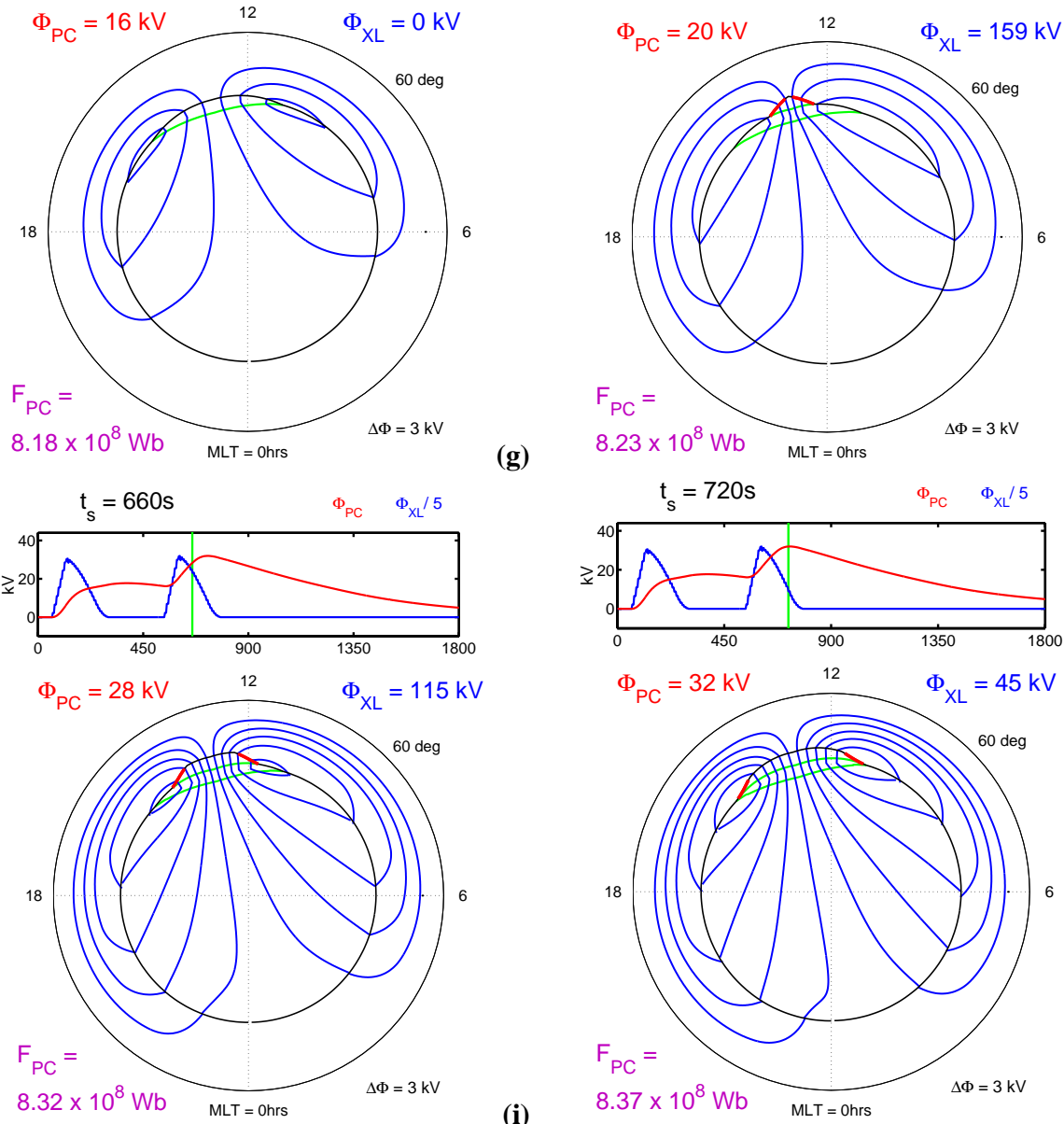

(i)
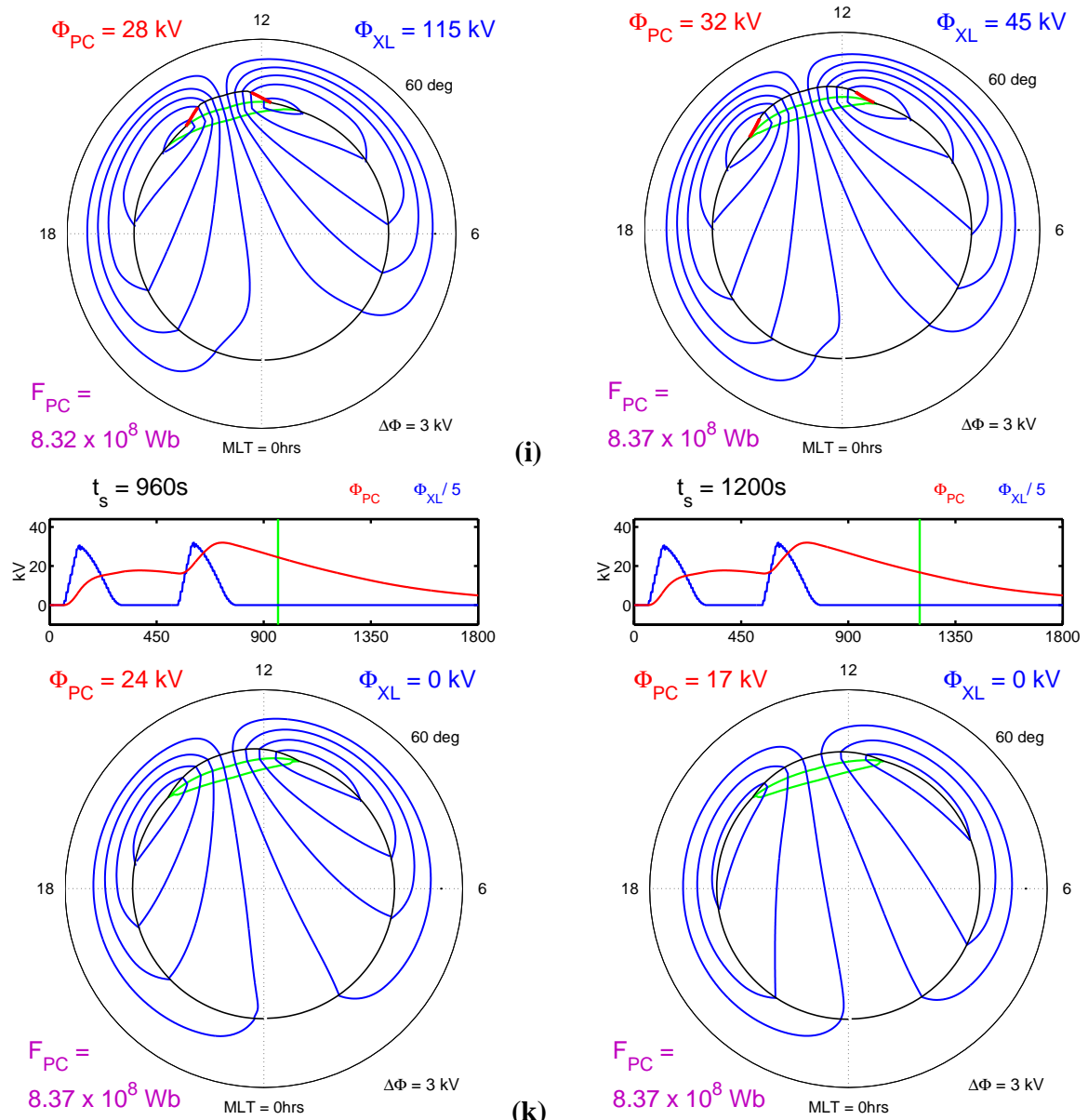

(h)

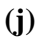

(k)

(l)

Fig. 7. Continued. 

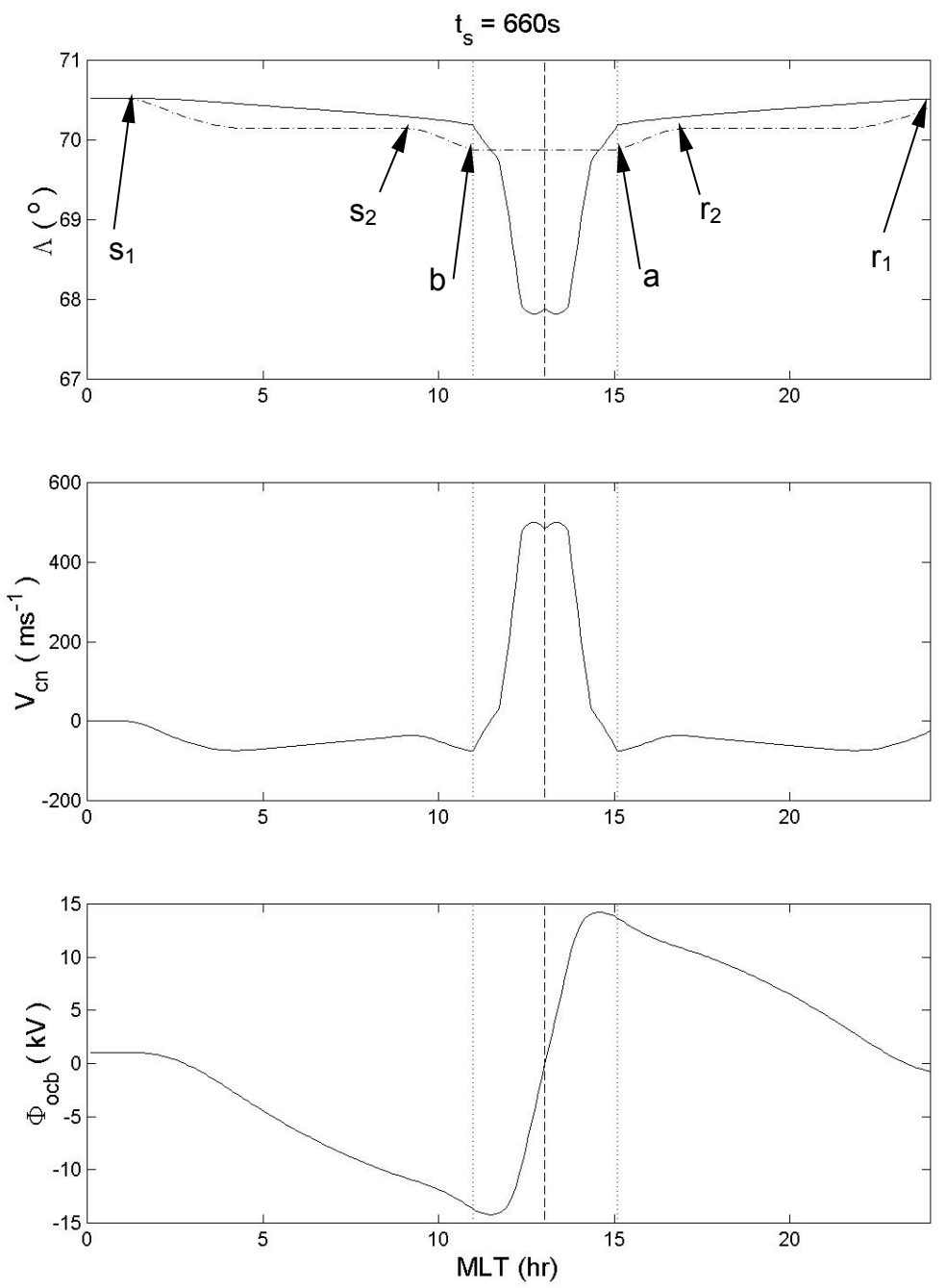

Fig. 8. Same as Fig. 4 but for simulation time $t_{s}=660 \mathrm{~s}$. Note that the vertical axes scales are expanded compared to those used in Figs. 4 and 5 .

the equatorward motions of the boundary seen during transient events by ground-based radars and optical imagers (e.g. Lockwood et al., 1993a, b; Milan et al., 2000) and spacebased proton aurora imagers (Lockwood et al., 2003). It is also consistent with the separation of cusp ion step signatures seen by both low and middle altitude spacecraft (e.g. Lockwood and Davis, 1996).

\section{Discussion}

The results presented in the previous section reveal some interesting features of the convection response to reconnection pulses and, in particular, how that response depends upon the pre-existing flow that remains following prior reconnection activity. The principles behind this can be understood from Fig. 8, which is the plot equivalent to Fig. 4, but for simulation time $t_{s}=660 \mathrm{~s}$ corresponding to Fig. 7i. The top panel shows the latitudes of the OCB and the equilibrium boundaries $\left(\Lambda_{\mathrm{OCB}}\right.$ and $\left.\Lambda_{E}\right)$; the effects of both of the reconnection pulses can be seen in both boundaries. The perturbation to the equilibrium boundary, caused by the first pulse, has reached 24:00 MLT on the dusk side $\left(r_{1}\right)$ and almost 1:00 MLT on the dawnside $\left(s_{1}\right)$. Thus, this perturbation due to the first pulse is close to encircling the entire polar cap, after which the flow would have, in the absence of the second pulse, decayed exponentially in a quasi shape-preserving manner. However, the perturbation due to the second pulse can be seen emerging from the merging gap (with $r_{2}$ propagating east, away from $a$, and $s_{2}$ propagating west away from $b)$. The effect of the first pulse can be seen in the OCB latitude by the fact that it has moved equatorward at MLTs outside the merging gap (except near midnight, where neither $\Lambda_{E}$ nor $\Lambda_{\mathrm{OCB}}$ has yet to be influenced). The effect of the second pulse can be seen as a large equatorward migration within the merging gap ab. The two pulses can be identified in the poleward convection speeds, with equatorward motion $\left(V_{c n}<0\right)$ at MLTs outside the merging gap (except near mid- 
night) and strong poleward flow $\left(V_{c n}>0\right)$ inside the merging gap. An enhancement of the equatorward flow is seen just outside of the merging gap (between $a$ and $r_{2}$ and between $b$ and $s_{2}$ ) but this is much weaker than the poleward flow between $a$ and $b$. This should be contrasted with the situation for the equivalent time following the first pulse where the total equatorward flow (between $a$ and $s_{1}$ and between $b$ and $r_{1}$ ) matches the total poleward flow between $a$ and $b$. Indeed, in the bottom panel of Fig. 8 it can be seen that the additional equatorward flow between a and $s_{2}$ and between $b$ and $r_{2}$ causes only a very small perturbation to the potential distribution around the OCB.

The important point is that, at $s_{2}$ and $r_{2}$, the OCB and the equilibrium boundaries are close together, such that $\left|\Lambda_{\mathrm{OCB}}-\Lambda_{E}\right|$ is small and thus $V_{c n}$ is near zero. This situation arises because the equilibrium boundary at these MLTs is perturbed equatorward by the second pulse, while the OCB has been perturbed equatorward by a similar amount by the first pulse. This means that the flow streamlines that flow poleward into the polar cap between $a$ and $b$ flow back equatorward across the OCB, not sunward of $s_{2}$ and $r_{2}$, as would have been the case without the prior reconnection pulse, but closer to midnight. Near $s_{2}$ and $r_{2}$ at this $t_{s}$, the flow that would have been caused by the first pulse in isolation is poleward whereas that which would be caused by the second pulse in isolation would be equatorward and comparable in magnitude. Thus they almost completely cancel each other out. The net effect is that the flows due to the two pulses add to form the flow pattern given in Fig. 7i, which shows enhancement at all MLT (except near midnight) in response to the second reconnection pulse. Thus the response is much more global in nature than it was for the first pulse. A full analysis and comparison of the expanding and quasiglobal responses will be presented in a later paper (Morley and Lockwood, 2003).

The two reconnection pulses in the sequence simulated here are identical and the differences in the response of the second pulse are entirely due to the remnant effects of the first pulse. Thus this example, for the first time, highlights the importance of the reconnection history in determining the response to a given reconnection voltage change. This factor has not previously been considered and may offer an explanation of the different convection responses to southward turnings of the IMF that have been reported in the literature (see Sect. 1.2). Indeed, Fig. 7 suggests that the response to the second pulse is more instantaneous in nature, whereas that following the first pulse is clearly expanding in nature. This possibility will be investigated in detail in a follow-up publication.

\section{Applications of the model}

The numerical model presented here has many applications, beyond the studies of the convection response to reconnection rate variations, of which we have presented just one example. In this paper we have concentrated on variations in the dayside magnetopause reconnection rate. This case is relatively simple because the associated precipitation in the cusp is relatively soft and conductivity changes are not as important a factor as they are on the nightside where precipitation has higher energies. The model presented here does not include spatial structure in the ionospheric conductivities and, in general, these will influence the pattern of convection. Allowance for this would require a much more complex algorithm than is used here to convert the potential distribution around the OCB into an equipotential map of everywhere poleward of the region 2 field-aligned current ring. However, extensions to the model which made allowance for the spatial and temporal variations of precipitation would improve the realism of the model and would increase the number of applications.

Conductivity structure and variations become a highly relevant factor when discussing the nightside and, in particular, the phenomenon of substorms. For computing the potential distribution around the OCB, the model can readily be generalised to include nightside reconnection in the cross-tail current sheet. However, the precipitation associated with substorms generates conductivity structure which varies rapidly at substorm onset.

For the dayside reconnection phenomena, a large number of studies are made possible by the model in its present form, because precipitation down newly-opened field lines generates a number of signatures and the nature of those signatures depends on how those field lines are moved by convection. Examples of this type of application include modelling cusp ion steps, cusp proton aurora bursts seen by global imagers, poleward-moving events seen by incoherent and coherent scatter radar, polar cap patches, red-line auroral transients and dayside ion heating events. In addition, in a future paper, we will apply the model to investigate the various methods that have been applied to observations to determine the rate of expansion of the flow pattern.

Acknowledgements. The authors thank Mervyn Freeman of British Antarctic Survey for many helpful discussions and to B.S. Lanchester of the University of Southampton for helpful discussions, proof reading and support. This work was supported by the UK Particle Physics and Astronomy Research Council.

Topical Editor M. Lester thanks two referees for their help in evaluating this paper.

\section{References}

Banks, P. M., Araki, T., Clauer, C. R., St-Maurice, J. P., and Foster, J. C.: The interplanetary magnetic field, cleft currents, and plasma convection in the polar caps, Planet. Space Sci., 32, 1551-1560, 1984.

Clauer, C. R. and Banks, P. M.: Relationship of interplanetary electric field to the high latitude ionospheric electric field and currents: observations and model simulation, J. Geophys. Res., 93, 2749-2754, 1988.

Cowley, S. W. H.: Solar wind control of magnetospheric convection, in: Achievements of the international magnetospheric 
study, IMS, pp 483-494, ESA SP-217, ESTEC, Noordwijk, The Netherlands, 1984

Cowley, S. W. H. and Lockwood, M.: Excitation and decay of solar wind driven flows in the magnetosphere-ionosphere system, Ann. Geophysicae, 10, 103-115, 1992.

Cowley, S. W. H. and Lockwood, M.: Incoherent scatter radar observations related to magnetospheric dynamics, Adv. Space Res., $20(4 / 5), 873-882,1997$.

Cowley, S. W. H., Khan, H., and Stockton-Chalk, A.: Plasma flow in the coupled magnetosphere-ionosphere system and its relationship to the substorm cycle, in: "Substorms 4", edited by Kokubun, S. and Kamide, Y., Terra. Sci, Tokyo, pp. 623-628, 1998.

Erlandson, R. E., Zanetti, L. J., Potemra, T. A., Bythrow, P. F., and Lundin, R.: IMF $B_{y}$ dependence of region 1 Birkeland currents near noon, J. Geophys. Res., 93, 9804, 1988.

Etemadi, A., Cowley, S. W. H., Lockwood, M., Bromage, B. J. I., Willis, D. M., and Lühr, H.: The dependence of high-latitude dayside ionospheric flows on the north-south component of the IMF: a high time resolution correlation analysis using EISCAT "POLAR" and AMPTE UKS and IRM data, Planet. Space Sci., 36, 471-498, 1988.

Etemadi, A., Cowley, S. W. H., and Lockwood, M.: The effect of rapid changes in ionospheric flow on velocity vectors deduced from radar beam-swinging experiments, J. Atmos. Terr. Phys., 51, 125-138, 1989.

Friis-Christensen, E., Kamide, Y., Richmond, A. D., and Matsushita, S.: Interplanetary magnetic field control of high-latitude electric fields and currents determined from Greenland magnetometer data, J. Geophys. Res., 90, 1325, 1985.

Freeman, M. P., Ruohoniemi, J. M., and Greenwald, R. A.: The determination of time-stationary two-dimensional convection patterns with single-station radar, J. Geophys. Res., 96, 15735$15749,1991$.

Greenwald, R. A., Ruohoniemi, J. M., Bristow, W. A., Sofko, G. J., Villain, J.-P., Huuskonen, A., Kokubun, S., and Frank, L. A.: Mesoscale dayside convection vortices and their relation to substorm phase, J. Geophys. Res., 101, 21 607-21 702, 1996.

Hairston, M. R. and Heelis, R. A.: Model of the high-latitude ionospheric convection pattern during southward Interplanetary Magnetic Field using DE 2 data, J. Geophys. Res., 95, 2333, 1990.

Heelis, R. A., Lowell, J. K., and Spiro, R. W.: A model of the high-latitude ionospheric convection pattern, J. Geophys. Res., 87, 6339, 1982.

Heppner, J. P. and Maynard, N. C.: Empirical high-latitude electric field models, J. Geophys. Res., 92, 4467, 1987.

Holt, J. M., Ward, R. H., Evans, J. V., and Oliver, W. L.: Empirical models for the plasma convection at high latitudes from Millstone Hill observations, J. Geophys. Res., 92, 203, 1987.

Iijima, T., Fujii, R., Potemra, T. A., and Saflekos, N. A.: Fieldaligned currents in the south polar cusp and their relationship to the interplanetary magnetic field, J. Geophys. Res., 83, 5595, 1978.

Khan, H. and Cowley, S. W. H.: Observations of the response time of high-latitude ionospheric convection to variations in the interplanetary field using EISCAT and IMP-8 data, Ann. Geophysicae, 17, 1306-1355, 1999.

Knipp, D. J., Richmond, A. D., Emery, B., Crooker, N. U., de la Beaujardiere, O., Evans, D., and Kroehl, H.: Ionospheric convection response to changing IMF direction, Geophys. Res. Lett., 18, 721-724, 1991.

Lockwood, M.: Ionospheric signatures of pulsed magnetopause re- connection, in: "Physical signatures of magnetopause boundary layer Processes", edited by Holtet, J. A. and Egeland, A., NATO ASI Ser. C, 425, 229-243, 1994.

Lockwood, M. and Cowley, S. W.: Ionospheric convection and the substorm cycle, in: "Substorms 1, Proceedings of the First International Conference on Substorms, ICS-1", edited by Mattock, C., ESA-SP-335, 99-110, European Space Agency Publications, Nordvijk, The Netherlands, 1990.

Lockwood, M. and Cowley, S. W. H.: Comment on "Ionospheric convection response to changing IMF direction" by Knipp et al., Geophys. Res. Lett., 18, 2173-2174, 1991.

Lockwood, M. and Cowley, S. W. H.: Comment on "A statistical study of the ionospheric convection response to changing interplanetary magnetic field conditions using the assimilative mapping of ionospheric electrodynamics technique", J. Geophys. Res., 104, 4387-4391, 1999.

Lockwood, M. and Davis, C. J.: On the longitudinal extent of magnetopause reconnection bursts, Ann. Geophysicae, 14, 865-878, 1996.

Lockwood, M., van Eyken, A. P., Bromage, B. J. I., Willis, D. M., and Cowley, S. W. H.: Eastward propagation of a plasma convection enhancement following a southward turning of the interplanetary magnetic field, Geophys. Res. Lett., 13, 72-75, 1986.

Lockwood, M., Cowley, S. W. H., and Freeman, M. P.: The excitation of plasma convection in the high latitude ionosphere, J. Geophys Res., 95, 7961-7971, 1990.

Lockwood, M., Moen, J., Cowley, S. W. H., Farmer, A. D., Løvhaug, U. P., Lühr, H., and Davda, V. N.: Variability of dayside convection and motions of the cusp/cleft aurora, Geophys. Res. Lett., 20, 1011-1014, 1993a.

Lockwood, M., Denig, W. F., Farmer, A. D., Davda, V. N., Cowley, S. W. H., and Lühr, H.: Ionospheric signatures of pulsed magnetic reconnection at the Earth's magnetopause, Nature, 361 (6411), 424-428, 1993b.

Lockwood, M., Wild, M. N., and Cowley, S. W. H.: The Onset and Expansion of Enhanced Ionospheric Convection following a southward turning of the IMF, RAL report, 1999.

Lockwood, M., Davis, C. J., Onsager, T. G., and Scudder, J. A.: Modelling signatures of pulsed magnetopause reconnection in cusp ion dispersion signatures seen at middle altitudes, Geophys. Res. Lett., 25, 591-594, 1998.

Lockwood, M., Lanchester, B. S., Frey, H., Throp, K., Morley, S., Milan, S. E., and Lester, M. E.: IMF Control of Cusp Proton Emission Intensity and Dayside Convection: implications for component and anti-parallel reconnection., Ann. Geophysicae, 21, 955-982, 2003.

Lu, G., Reiff, P. H., Hairston, M. R., Heelis, R. A., and Karty, J. L.: Distribution of convection potential around the polar cap boundary as a function of Interplanetary magnetic field, J. Geophys. Res., 94, 13 447-13 461, 1989.

Lu, G., Richmond, A. D., Ruohoniemi, J. M., Greenwald, R. A., Hairston, M., Rich, F. J., and Evans, D. S.: An investigation of the influence of data and model inputs on assimilative mapping of ionospheric electrodynamics, J. Geophys. Res., 106, 417-433, 2001.

Lu, G., Cowley, S. W. H., Milan, S. E., Sibeck, D. G., Greenwald, R. A., and Moretto, T.: Solar wind effects on ionospheric convection: a review, J. Atmos. S.-P., 64, 145-157, 2002.

McWilliams, K. A., Yeoman, T. K., Sigwarth, J. B., Frank, L. A., and Brittnacher, M.: The dayside ultraviolet aurora and convection responses to a southward turning of the interplanetary magnetic field, Ann. Geophysicae, 19, 707-721, 2001. 
Milan, S. E., Lester, M., Cowley, S. W. H., and Brittnacher, M.: Convection and auroral response to a southward turning of the IMF: Polar UVI, CUTLASS and IMAGE signatures of transient magnetic flux transfer at the magnetopause, J. Geophys. Res., 105, 15 741-15 755, 2000.

Morley, S. K. and Lockwood, M.: A numerical model of the ionospheric signatures of time-varying magnetic reconnection: II. Quasi-instantaneous and expanding responses to IMF changes in ionospheric convection and ion heating, Ann. Geophysicae, to be submitted, 2003.

Murr, D. L. and Hughes, W. J.: Reconfiguration timescales of ionospheric convection, Geophys. Res. Lett., 28, 2145-2148, 2001.

Nishida, A.: Coherence of geomagnetic DP 2 fluctuations with interplanetary magnetic field variations, J. Geophys. Res., 73, 5549-5559, 1968a.

Nishida, A.: Geomagnetic DP 2 fluctuations and associated magnetospheric phenomena, J. Geophys. Res., 73, 1795, 1968 b.

Reiff, P. H. and Burch, J. L.: $B_{y}$-dependent dayside plasma flow and Birkeland currents in the dayside magnetosphere: 2. A global model for northward and southward IMF, J. Geophys. Res., 90, 1595-1609, 1985.

Ridley, A. J., Clauer, C. R., Lu, G., and Papitashvilli, V. O.: Ionospheric convection during nonsteady interplanetary magnetic field conditions, J. Geophys. Res., 102, 14563-14573, 1997.

Ridley, A. J., Lu, G., Clauer, C. R., and Papitashvilli, V. O.: A statistical study of the ionospheric convection response to changing interplanetary magnetic field conditions using the assimilative mapping of ionospheric electrodynamics technique, J. Geophys. Res., 103, 4023-4039, 1998.

Ridley, A. J., Lu, G., Clauer, C. R., and Papitashvilli, V. O.: Reply, J. Geophys. Res., 104, 4393-4396, 1999.
Ruohoniemi, J. M. and Baker, K. L.: Large-scale imaging of highlatitude convection with Super Dual Auroral Radar Network HF radar observation, J. Geophys. Res., 103, $20797,1998$.

Ruohoniemi, J. M. and Greenwald, R. A.: The response of high latitude convection to a sudden southward IMF turning, Geophys. Res. Lett., 25, 2913-2916, 1998.

Ruohoniemi, J. M., Shepherd, S. G., and Greenwald, R. A.: The response of the high-latitude ionosphere to IMF variations, J. Atmos. S.-P., 64, 159-171, 2002.

Saunders, M. A., Freeman, M. P., Southwood, D. J., Cowley, S. W. H., Lockwood, M., Samson, J. C., Farrugia, C. J., and Hughes, T. J.: Dayside ionospheric convection changes in response to long period IMF oscillations: determination of the ionospheric phase velocity, J. Geophys. Res., 97, 19373-19380, 1992.

Siscoe, G. L. and Huang, T. S.: Polar cap inflation and deflation, J. Geophys. Res., 90, 543, 1985.

Slinker, S. P., Fedder, J. A., Ruohoniemi, J. M., and Lyon, J. G.: Global MHD simulation of the magnetosphere for Nov. 24, 1966 J. Geophys. Res., 106, 361-380, 2000.

Spreiter, J. R., Summers, A. L., and Alksne, A. Y.: Hydromagnetic flow around the magnetosphere, Planet. Space Sci., 14, 223-253, 1966.

Smith, M. F. and Lockwood, M.: The Earth's magnetospheric cusps, Rev. Geophys., 34 (2), 233-260, 1996.

Todd, H., Cowley, S. W. H., Lockwood, M., Willis, D. M., and Lühr, H.: Response time of the high-latitude dayside ionosphere to sudden changes in the north-south component of the IMF, Planet. Space Sci., 36, 1415-1428, 1988.

Weimer, D. R.: Models of high-latitude electric potentials derived with a least-error fit spherical harmonic coefficients, J. Geophys. Res.,100, 19595-19608, 1995. 